\title{
Comparison of Fragmentation Techniques for the Structural Characterisation of Singly Charged Agrochemicals
}

by

Bryan P. Marzullo ${ }^{1}$, Tomos E. Morgan ${ }^{1}$, Christopher A. Wootton ${ }^{1}$, Meng $\mathrm{Li}^{1}$, Simon J. Perry ${ }^{2}$, Mansoor Saeed ${ }^{2}$, Mark P. Barrow ${ }^{1}$, and Peter B. O'Connor*,1

1. Department of Chemistry, University of Warwick, Coventry, CV4 7AL;

2. Syngenta, Jealott's Hill International Research Centre, Product Metabolism \& Analytical Sciences, RG42 6EY

Corresponding authors: Peter O'Connor p.oconnor@warwick.ac.uk

Contents

Table S 1: Table showing the peak list, relative intensity, assignments, and er rors (ppm) of the CAD fragmentation of Azoxystrobin (Figure S 2a).

Table S 2: Table showing the peak list, relative intensity, assignments, and errors (ppm) of the EID fragmentation of Azoxystrobin (Figure $S 2 b$ ).

Table S 3: Table showing the peak list, relative intensity, assignments, and errors (ppm) of the CAD fragmentation of Fenpropidin (Figure 1a).

Table S 4: Table showing the peak list, relative intensity, assignments, and errors (ppm) of the EID MS/MS of Fenpropidin (Figure $1 \mathrm{~b}$ ).

Table S 5: Table showing the peak list, relative intensity, assignments, and errors (ppm) of the CAD fragmentation of Fluazifop acid (Figure 3a).

Table S 6: Table showing the peak list, relative intensity, assignments, and errors (ppm) of the EID fragmentation of Fluazifop acid (Figure $3 b$ ).

Table S 7: Table showing the peak list, relative intensity, assignments, and errors (ppm) of the CAD fragmentation of Fluazifop-p-butyl (Figure 4a).

Table S 8: Table showing the peak list, relative intensity, assignments, and errors (ppm) of the EID fragmentation of Fluazifop-p-butyl (Figure 4b).

Table S 9: Table showing the peak list, relative intensity, assignments, and errors (ppm) of the CAD fragmentation of Nicosulfuron (Figure $S 1$ a).

Table S 10: Table showing the peak list, relative intensity, assignments, and errors (ppm) of the EID fragmentation of Nicosulfuron (Figure $S 1 b$ ).

Table S 11: Table showing the peak list, relative intensity, assignments, and errors (ppm) of the CAD fragmentation of Pirimiphos-methyl (Figure 2a).

Table S 12: Table showing the peak list, relative intensity, assignments, and errors (ppm) of the EID fragmentation of Pirimiphos-methyl (Figure $2 b$ ).

Table S 13: Table showing the peak list, relative intensity, assignments, and errors (ppm) of the CAD fragmentation of s-metolachlor (Figure S 3a).

Table S 14: Table showing the peak list, relative intensity, assignments, and errors (ppm) of the EID fragmentation of s-metolachlor (Figure $S 3 b$ ).

Table S 15: Table showing the percentage of even and odd electron fragments for both fragmentation methods (CAD and EID)

Figure S 1: The fragmentation spectra of nicosulfuron along with the corresponding cleavage diagram via a) CAD (20 V) and b) EID (18 V). Peak assignment tables are displayed in Table S9 \& S10. 
Figure S 2: The fragmentation spectra of s-metolachlor with its corresponding cleavage assignment diagram via a) CAD (20 V) and b) EID (18 V). Peak assignment are listed in Tables S13 \& S14.

Figure S 3: Zoom in view of the fluazifop acid CAD spectrum showing the separation of the D cleavage $\left(\mathrm{C}_{12} \mathrm{H}_{7} \mathrm{~F}_{3} \mathrm{NO}_{2}\right)$ and the methyl rearrangement $\left(\mathrm{C}_{13} \mathrm{H}_{11} \mathrm{~F}_{3} \mathrm{NO}\right)$ peaks observed at nominal mass of $\mathrm{m} / \mathrm{z} 254$ and their corresponding isotope peaks labelled with a triangle and a square, respectively. Also in this zoomed in spectrum, separation of the isotope peaks and a peak corresponding to a composition of $\mathrm{C}_{12} \mathrm{H}_{8} \mathrm{~F}_{3} \mathrm{NO}_{2}$ can be observed.

Figure S 4: Isolation spectra for the 7 agrochemicals: A) azoxystrobin, B) fenpropidin, C) fluazifop acid, D) fluazifop-p-butyl, E) nicosulfuron, F) pirimiphos-methyl, and G) S-metolachlor. 
Table S 1: Table showing the peak list, relative intensity, assignments, and errors (ppm) of the CAD fragmentation of Azoxystrobin (Figure S 2a). ${ }^{*}$ displays the peaks that were used for the internal calibration.

\begin{tabular}{|c|c|c|c|c|c|c|c|}
\hline & $m / z$ & $\begin{array}{l}\text { Relative } \\
\text { Intensity }\end{array}$ & $S / N$ & $\begin{array}{l}\text { Elemental } \\
\text { Composition }\end{array}$ & $\begin{array}{c}\text { Theoretical } \\
\text { Mass }\end{array}$ & $\begin{array}{l}\text { Error } \\
\text { (ppm) }\end{array}$ & Assignments \\
\hline & 404.1236 & 0.02 & 12 & $\mathrm{C}_{22} \mathrm{H}_{18} \mathrm{~N}_{3} \mathrm{O}_{5}$ & 404.1241 & -1.121 & {$[\mathrm{M}+\mathrm{H}]^{+}$} \\
\hline \multirow[t]{6}{*}{$*$} & 372.0979 & 100 & 21614 & $\mathrm{C}_{21} \mathrm{H}_{14} \mathrm{~N}_{3} \mathrm{O}_{4}$ & 372.0979 & -0.014 & A \\
\hline & 344.1029 & 5.21 & 13700 & $\mathrm{C}_{20} \mathrm{H}_{14} \mathrm{~N}_{3} \mathrm{O}_{3}$ & 344.103 & -0.215 & B \\
\hline & 340.0716 & 0.07 & 129 & $\mathrm{C}_{20} \mathrm{H}_{10} \mathrm{~N}_{3} \mathrm{O}_{3}$ & 340.0717 & -0.191 & AA \\
\hline & 329.0795 & 0.36 & 4556 & $\mathrm{C}_{19} \mathrm{H}_{11} \mathrm{~N}_{3} \mathrm{O}_{3}$ & 329.0795 & -0.128 & AC \\
\hline & 328.0717 & 0.03 & 210 & $\mathrm{C}_{19} \mathrm{H}_{10} \mathrm{~N}_{3} \mathrm{O}_{3}$ & 328.0717 & 0.026 & \\
\hline & 317.0921 & 0.62 & 1091 & $\mathrm{C}_{19} \mathrm{H}_{13} \mathrm{~N}_{2} \mathrm{O}_{3}$ & 317.0921 & -0.047 & $\mathrm{BD} / \mathrm{BE}$ \\
\hline \multirow[t]{6}{*}{$*$} & 316.108 & 2.47 & 5101 & $\mathrm{C}_{19} \mathrm{H}_{14} \mathrm{~N}_{3} \mathrm{O}_{2}$ & 316.1081 & -0.070 & $A B$ \\
\hline & 315.0764 & 0.16 & 362 & $\mathrm{C}_{19} \mathrm{H}_{11} \mathrm{~N}_{2} \mathrm{O}_{3}$ & 315.0764 & -0.135 & $\mathrm{AAD} / \mathrm{BE}$ \\
\hline & 312.0767 & 0.09 & 301 & $\mathrm{C}_{19} \mathrm{H}_{10} \mathrm{~N}_{3} \mathrm{O}_{2}$ & 312.0768 & -0.100 & $A B$ \\
\hline & 303.0764 & 1.17 & 2169 & $\mathrm{C}_{18} \mathrm{H}_{11} \mathrm{~N}_{2} \mathrm{O}_{3}$ & 303.0764 & -0.057 & $A C D$ \\
\hline & 302.0812 & 0.02 & 64 & $\mathrm{C}_{19} \mathrm{H}_{12} \mathrm{NO}_{3}$ & 302.0812 & 0.030 & $\mathrm{BH}$ \\
\hline & 289.0971 & 0.08 & 292 & $\mathrm{C}_{18} \mathrm{H}_{13} \mathrm{~N}_{2} \mathrm{O}_{2}$ & 289.0972 & -0.032 & $\mathrm{ABD} / \mathrm{ABE}$ \\
\hline \multirow[t]{6}{*}{$*$} & 287.0815 & 0.56 & 1639 & $\mathrm{C}_{18} \mathrm{H}_{11} \mathrm{~N}_{2} \mathrm{O}_{2}$ & 287.0815 & -0.013 & $\mathrm{ABD} / \mathrm{ABE}$ \\
\hline & 275.0815 & 0.01 & 51 & $\mathrm{C}_{17} \mathrm{H}_{11} \mathrm{~N}_{2} \mathrm{O}_{2}$ & 275.0815 & 0.089 & $\mathrm{BCD} / \mathrm{BCE}$ \\
\hline & 274.0863 & 0.13 & 300 & $\mathrm{C}_{18} \mathrm{H}_{12} \mathrm{NO}_{2}$ & 274.0863 & -0.005 & \\
\hline & 202.0499 & 0.02 & 52 & $\mathrm{C}_{11} \mathrm{H}_{8} \mathrm{NO}_{3}$ & 202.0499 & 0.074 & $A G$ \\
\hline & 201.0659 & 0.09 & 283 & $\mathrm{C}_{11} \mathrm{H}_{9} \mathrm{~N}_{2} \mathrm{O}_{2}$ & 201.0659 & 0.021 & $\mathrm{BF} / \mathrm{ACM}$ \\
\hline & 199.0502 & 0.02 & 47 & $\mathrm{C}_{11} \mathrm{H}_{7} \mathrm{~N}_{2} \mathrm{O}_{2}$ & 199.0502 & -0.019 & $\mathrm{BF} / \mathrm{BCl}$ \\
\hline \multirow[t]{2}{*}{$*$} & 197.0709 & 0.02 & 84 & $\mathrm{C}_{12} \mathrm{H}_{9} \mathrm{~N}_{2} \mathrm{O}$ & 197.0709 & 0.001 & $A B M$ \\
\hline & 172.0393 & 0.22 & 514 & $\mathrm{C}_{10} \mathrm{H}_{6} \mathrm{NO}_{2}$ & 172.0393 & -0.181 & $\mathrm{~J}^{\prime} \mathrm{H}$ \\
\hline & & & & & $\begin{array}{l}\text { Standard } \\
\text { Deviation }\end{array}$ & 0.086 & \\
\hline & & & & & $\begin{array}{l}\text { Absolute } \\
\text { Average }\end{array}$ & 0.072 & \\
\hline
\end{tabular}


Table S 2: Table showing the peak list, relative intensity, assignments, and errors (ppm) of the EID fragmentation of Azoxystrobin (Figure S $2 b$ ). ${ }^{*}$ displays the peaks that were used for the internal calibration.

\begin{tabular}{|c|c|c|c|c|c|c|c|}
\hline & $\mathrm{m} / \mathrm{z}$ & $\begin{array}{c}\text { Relative } \\
\text { intensity \% }\end{array}$ & $S / N$ & $\begin{array}{l}\text { Elemental } \\
\text { Composition }\end{array}$ & $\begin{array}{c}\text { Theoretical } \\
\text { Mass }\end{array}$ & $\begin{array}{l}\text { Error } \\
\text { (ppm) }\end{array}$ & Assignments \\
\hline & 404.12414 & 100.00 & 15501 & $\mathrm{C}_{22} \mathrm{H}_{18} \mathrm{~N}_{3} \mathrm{O}_{5}$ & 404.12410 & 0.106 & {$[\mathrm{M}+\mathrm{H}]^{+}$} \\
\hline \multirow[t]{30}{*}{$*$} & 388.09279 & 0.10 & 13 & $\mathrm{C}_{21} \mathrm{H}_{14} \mathrm{~N}_{3} \mathrm{O}_{5}$ & 388.09280 & -0.008 & $\mathrm{R}$ \\
\hline & 372.09796 & 17.98 & 2821 & $\mathrm{C}_{21} \mathrm{H}_{14} \mathrm{~N}_{3} \mathrm{O}_{4}$ & 372.09788 & 0.213 & A \\
\hline & 371.09008 & 0.11 & 16 & $\mathrm{C}_{21} \mathrm{H}_{13} \mathrm{~N}_{3} \mathrm{O}_{4}$ & 371.09006 & 0.070 & \\
\hline & 357.07449 & 1.48 & 231 & $\mathrm{C}_{20} \mathrm{H}_{11} \mathrm{~N}_{3} \mathrm{O}_{4}$ & 357.07441 & 0.232 & \\
\hline & 344.10307 & 4.63 & 734 & $\mathrm{C}_{20} \mathrm{H}_{14} \mathrm{~N}_{3} \mathrm{O}_{3}$ & 344.10297 & 0.300 & B \\
\hline & 340.07175 & 3.19 & 507 & $\mathrm{C}_{20} \mathrm{H}_{10} \mathrm{~N}_{3} \mathrm{O}_{3}$ & 340.07167 & 0.241 & \\
\hline & 330.08747 & 0.11 & 15 & $\mathrm{C}_{19} \mathrm{H}_{12} \mathrm{~N}_{3} \mathrm{O}_{3}$ & 330.08732 & 0.448 & \\
\hline & 329.07958 & 4.25 & 680 & $\mathrm{C}_{19} \mathrm{H}_{11} \mathrm{~N}_{3} \mathrm{O}_{3}$ & 329.07949 & 0.256 & $A C$ \\
\hline & 328.10819 & 0.39 & 61 & $\mathrm{C}_{20} \mathrm{H}_{14} \mathrm{~N}_{3} \mathrm{O}_{2}$ & 328.10805 & 0.405 & \\
\hline & 328.07174 & 1.63 & 259 & $\mathrm{C}_{19} \mathrm{H}_{10} \mathrm{~N}_{3} \mathrm{O}_{3}$ & 328.07167 & 0.215 & \\
\hline & 326.09254 & 0.25 & 39 & $\mathrm{C}_{20} \mathrm{H}_{12} \mathrm{~N}_{3} \mathrm{O}_{2}$ & 326.09240 & 0.435 & \\
\hline & 317.09219 & 1.14 & 182 & $\mathrm{C}_{19} \mathrm{H}_{13} \mathrm{~N}_{2} \mathrm{O}_{3}$ & 317.09207 & 0.377 & $B D$ \\
\hline & 316.10817 & 2.55 & 408 & $\mathrm{C}_{19} \mathrm{H}_{14} \mathrm{~N}_{3} \mathrm{O}_{2}$ & 316.10805 & 0.380 & $A B$ \\
\hline & 315.10038 & 0.14 & 20 & $\mathrm{C}_{19} \mathrm{H}_{13} \mathrm{~N}_{3} \mathrm{O}_{2}$ & 315.10023 & 0.482 & \\
\hline & 315.07650 & 0.24 & 36 & $\mathrm{C}_{19} \mathrm{H}_{11} \mathrm{~N}_{2} \mathrm{O}_{3}$ & 315.07642 & 0.266 & $\mathrm{BE}$ \\
\hline & 314.09253 & 0.20 & 30 & $\mathrm{C}_{19} \mathrm{H}_{12} \mathrm{~N}_{3} \mathrm{O}_{2}$ & 314.09240 & 0.411 & $A B$ \\
\hline & 313.06085 & 0.57 & 89 & $\mathrm{C}_{19} \mathrm{H}_{9} \mathrm{~N}_{2} \mathrm{O}_{3}$ & 313.06077 & 0.273 & $\mathrm{BE}$ \\
\hline & 312.07683 & 0.90 & 142 & $\mathrm{C}_{19} \mathrm{H}_{10} \mathrm{~N}_{3} \mathrm{O}_{2}$ & 312.07675 & 0.248 & $A B$ \\
\hline & 311.06908 & 0.15 & 22 & $\mathrm{C}_{19} \mathrm{H}_{9} \mathrm{~N}_{3} \mathrm{O}_{2}$ & 311.06893 & 0.504 & \\
\hline & 303.07649 & 2.06 & 331 & $\mathrm{C}_{18} \mathrm{H}_{11} \mathrm{~N}_{2} \mathrm{O}_{3}$ & 303.07642 & 0.225 & $A C D$ \\
\hline & 302.06879 & 0.13 & 19 & $\mathrm{C}_{18} \mathrm{H}_{10} \mathrm{~N}_{2} \mathrm{O}_{3}$ & 302.06859 & 0.654 & \\
\hline & 301.09722 & 0.30 & 47 & $\mathrm{C}_{19} \mathrm{H}_{13} \mathrm{~N}_{2} \mathrm{O}_{2}$ & 301.09715 & 0.213 & \\
\hline & 301.08465 & 0.83 & 134 & $\mathrm{C}_{18} \mathrm{H}_{11} \mathrm{~N}_{3} \mathrm{O}_{2}$ & 301.08458 & 0.239 & \\
\hline & 300.11318 & 0.07 & 10 & $\mathrm{C}_{19} \mathrm{H}_{14} \mathrm{~N}_{3} \mathrm{O}$ & 300.11314 & 0.136 & \\
\hline & 300.07682 & 0.83 & 133 & $\mathrm{C}_{18} \mathrm{H}_{10} \mathrm{~N}_{3} \mathrm{O}_{2}$ & 300.07675 & 0.215 & $\mathrm{CB}$ \\
\hline & 299.08147 & 0.10 & 14 & $\mathrm{C}_{19} \mathrm{H}_{11} \mathrm{~N}_{2} \mathrm{O}_{2}$ & 299.08150 & -0.105 & \\
\hline & 298.09777 & 0.07 & 10 & $\mathrm{C}_{19} \mathrm{H}_{12} \mathrm{~N}_{3} \mathrm{O}$ & 298.09749 & 0.940 & \\
\hline & 296.08201 & 0.15 & 22 & $\mathrm{C}_{19} \mathrm{H}_{10} \mathrm{~N}_{3} \mathrm{O}$ & 296.08184 & 0.582 & \\
\hline & 289.09729 & 0.19 & 28 & $\mathrm{C}_{18} \mathrm{H}_{13} \mathrm{~N}_{2} \mathrm{O}_{2}$ & 289.09715 & 0.461 & $A B D$ \\
\hline & 288.11318 & 0.11 & 16 & $\mathrm{C}_{18} \mathrm{H}_{14} \mathrm{~N}_{3} \mathrm{O}$ & 288.11314 & 0.149 & \\
\hline \multirow[t]{8}{*}{$*$} & 288.06552 & 0.13 & 19 & $\mathrm{C}_{18} \mathrm{H}_{10} \mathrm{NO}_{3}$ & 288.06552 & 0.013 & $\mathrm{ACH}$ \\
\hline & 287.08160 & 1.54 & 249 & $\mathrm{C}_{18} \mathrm{H}_{11} \mathrm{~N}_{2} \mathrm{O}_{2}$ & 287.08150 & 0.330 & $A B D$ \\
\hline & 286.07384 & 0.12 & 17 & $\mathrm{C}_{18} \mathrm{H}_{10} \mathrm{~N}_{2} \mathrm{O}_{2}$ & 286.07368 & 0.579 & \\
\hline & 285.08984 & 0.09 & 13 & $\mathrm{C}_{18} \mathrm{H}_{11} \mathrm{~N}_{3} \mathrm{O}$ & 285.08966 & 0.618 & \\
\hline & 285.06599 & 0.10 & 14 & $\mathrm{C}_{18} \mathrm{H}_{9} \mathrm{~N}_{2} \mathrm{O}_{2}$ & 285.06585 & 0.481 & $A B D$ \\
\hline & 284.08191 & 0.18 & 28 & $\mathrm{C}_{18} \mathrm{H}_{10} \mathrm{~N}_{3} \mathrm{O}$ & 284.08184 & 0.265 & \\
\hline & 284.05806 & 0.07 & 10 & $\mathrm{C}_{18} \mathrm{H}_{8} \mathrm{~N}_{2} \mathrm{O}_{2}$ & 284.05803 & 0.126 & \\
\hline & 275.08163 & 0.20 & 31 & $\mathrm{C}_{17} \mathrm{H}_{11} \mathrm{~N}_{2} \mathrm{O}_{2}$ & 275.08150 & 0.445 & $B C D$ \\
\hline
\end{tabular}




\begin{tabular}{|c|c|c|c|c|c|c|c|}
\hline & $\mathrm{m} / \mathrm{z}$ & $\begin{array}{c}\text { Relative } \\
\text { intensity \% }\end{array}$ & $S / N$ & $\begin{array}{l}\text { Elemental } \\
\text { Composition }\end{array}$ & $\begin{array}{c}\text { Theoretical } \\
\text { Mass }\end{array}$ & $\begin{array}{l}\text { Error } \\
\text { (ppm) }\end{array}$ & Assignments \\
\hline & 274.08638 & 0.38 & 60 & $\mathrm{C}_{18} \mathrm{H}_{12} \mathrm{NO}_{2}$ & 274.08626 & 0.459 & $\mathrm{ABH}$ \\
\hline & 274.07383 & 0.10 & 15 & $\mathrm{C}_{17} \mathrm{H}_{10} \mathrm{~N}_{2} \mathrm{O}_{2}$ & 274.07368 & 0.562 & \\
\hline & 273.08978 & 0.22 & 33 & $\mathrm{C}_{17} \mathrm{H}_{11} \mathrm{~N}_{3} \mathrm{O}$ & 273.08966 & 0.413 & \\
\hline & 273.06597 & 0.50 & 80 & $\mathrm{C}_{17} \mathrm{H}_{9} \mathrm{~N}_{2} \mathrm{O}_{2}$ & 273.06585 & 0.437 & $B C D$ \\
\hline & 272.08193 & 0.39 & 62 & $\mathrm{C}_{17} \mathrm{H}_{10} \mathrm{~N}_{3} \mathrm{O}$ & 272.08184 & 0.339 & \\
\hline & 272.07058 & 0.08 & 11 & $\mathrm{C}_{18} \mathrm{H}_{10} \mathrm{NO}_{2}$ & 272.07061 & -0.080 & $\mathrm{ABH}$ \\
\hline & 271.08668 & 0.15 & 22 & $\mathrm{C}_{18} \mathrm{H}_{11} \mathrm{~N}_{2} \mathrm{O}$ & 271.08659 & 0.341 & \\
\hline & 260.07072 & 0.08 & 12 & $\mathrm{C}_{17} \mathrm{H}_{10} \mathrm{NO}_{2}$ & 260.07061 & 0.430 & $\mathrm{BCH}$ \\
\hline & 259.08670 & 0.19 & 30 & $\mathrm{C}_{17} \mathrm{H}_{11} \mathrm{~N}_{2} \mathrm{O}$ & 259.08659 & 0.423 & \\
\hline & 259.06287 & 0.18 & 28 & $\mathrm{C}_{17} \mathrm{H}_{9} \mathrm{NO}_{2}$ & 259.06278 & 0.333 & \\
\hline & 258.07885 & 0.16 & 25 & $\mathrm{C}_{17} \mathrm{H}_{10} \mathrm{~N}_{2} \mathrm{O}$ & 258.07876 & 0.329 & \\
\hline & 257.07105 & 0.14 & 21 & $\mathrm{C}_{17} \mathrm{H}_{9} \mathrm{~N}_{2} \mathrm{O}$ & 257.07094 & 0.418 & \\
\hline & 256.10949 & 0.22 & 35 & $\mathrm{C}_{16} \mathrm{H}_{16} \mathrm{O}_{3}$ & 256.10940 & 0.371 & \\
\hline & 256.08712 & 0.08 & 11 & $\mathrm{C}_{17} \mathrm{H}_{10} \mathrm{~N}_{3}$ & 256.08692 & 0.749 & \\
\hline & 255.10167 & 0.13 & 20 & $\mathrm{C}_{16} \mathrm{H}_{15} \mathrm{O}_{3}$ & 255.10157 & 0.381 & \\
\hline & 254.09382 & 0.24 & 39 & $\mathrm{C}_{16} \mathrm{H}_{14} \mathrm{O}_{3}$ & 254.09375 & 0.275 & \\
\hline & 254.06863 & 0.10 & 15 & $\mathrm{C}_{14} \mathrm{H}_{10} \mathrm{~N}_{2} \mathrm{O}_{3}$ & 254.06859 & 0.161 & \\
\hline & 245.07106 & 0.15 & 24 & $\mathrm{C}_{16} \mathrm{H}_{9} \mathrm{~N}_{2} \mathrm{O}$ & 245.07094 & 0.480 & \\
\hline & 244.08704 & 0.12 & 18 & $\mathrm{C}_{16} \mathrm{H}_{10} \mathrm{~N}_{3}$ & 244.08692 & 0.471 & \\
\hline & 244.07585 & 0.06 & 9 & $\mathrm{C}_{17} \mathrm{H}_{10} \mathrm{NO}$ & 244.07569 & 0.668 & \\
\hline & 239.04515 & 0.12 & 18 & $\mathrm{C}_{13} \mathrm{H}_{7} \mathrm{~N}_{2} \mathrm{O}_{3}$ & 239.04512 & 0.121 & AAI \\
\hline & 236.08320 & 0.06 & 9 & $\mathrm{C}_{16} \mathrm{H}_{12} \mathrm{O}_{2}$ & 236.08318 & 0.075 & \\
\hline & 231.09170 & 0.08 & 12 & $\mathrm{C}_{16} \mathrm{H}_{11} \mathrm{~N}_{2}$ & 231.09167 & 0.095 & \\
\hline & 229.07610 & 0.14 & 22 & $\mathrm{C}_{16} \mathrm{H}_{9} \mathrm{~N}_{2}$ & 229.07602 & 0.310 & \\
\hline & 224.04549 & 0.30 & 49 & $\mathrm{C}_{12} \mathrm{H}_{6} \mathrm{~N}_{3} \mathrm{O}_{2}$ & 224.04545 & 0.153 & \\
\hline & 223.07401 & 0.07 & 9 & $\mathrm{C}_{13} \mathrm{H}_{9} \mathrm{~N}_{3} \mathrm{O}$ & 223.07401 & -0.016 & \\
\hline & 222.06623 & 0.07 & 9 & $\mathrm{C}_{13} \mathrm{H}_{8} \mathrm{~N}_{3} \mathrm{O}$ & 222.06619 & 0.195 & \\
\hline & 216.06554 & 0.92 & 157 & $\mathrm{C}_{12} \mathrm{H}_{10} \mathrm{NO}_{3}$ & 216.06552 & 0.097 & \\
\hline & 212.03424 & 1.33 & 227 & $\mathrm{C}_{12} \mathrm{H}_{6} \mathrm{NO}_{3}$ & 212.03422 & 0.115 & \\
\hline & 211.05019 & 0.09 & 14 & $\mathrm{C}_{12} \mathrm{H}_{7} \mathrm{~N}_{2} \mathrm{O}_{2}$ & 211.05020 & -0.044 & DP \\
\hline & 210.06620 & 0.21 & 35 & $\mathrm{C}_{12} \mathrm{H}_{8} \mathrm{~N}_{3} \mathrm{O}$ & 210.06619 & 0.055 & \\
\hline & 210.04240 & 0.95 & 163 & $\mathrm{C}_{12} \mathrm{H}_{6} \mathrm{~N}_{2} \mathrm{O}_{2}$ & 210.04238 & 0.097 & $\mathrm{ACM}$ \\
\hline & 202.04987 & 0.07 & 10 & $\mathrm{C}_{11} \mathrm{H}_{8} \mathrm{NO}_{3}$ & 202.04987 & 0.016 & $A G$ \\
\hline & 201.06586 & 0.26 & 43 & $\mathrm{C}_{11} \mathrm{H}_{9} \mathrm{~N}_{2} \mathrm{O}_{2}$ & 201.06585 & 0.030 & $\mathrm{BF}$ \\
\hline & 201.04205 & 0.24 & 39 & $\mathrm{C}_{11} \mathrm{H}_{7} \mathrm{NO}_{3}$ & 201.04204 & 0.030 & \\
\hline$*$ & 200.03422 & 0.10 & 15 & $\mathrm{C}_{11} \mathrm{H}_{6} \mathrm{NO}_{3}$ & 200.03422 & -0.007 & AG \\
\hline & 199.05018 & 0.13 & 20 & $\mathrm{C}_{11} \mathrm{H}_{7} \mathrm{~N}_{2} \mathrm{O}_{2}$ & 199.05020 & -0.098 & $\mathrm{BF} / \mathrm{BCl}$ \\
\hline & 198.06749 & 0.06 & 9 & $\mathrm{C}_{13} \mathrm{H}_{10} \mathrm{O}_{2}$ & 198.06753 & -0.193 & \\
\hline & 197.13248 & 0.08 & 12 & $\mathrm{C}_{15} \mathrm{H}_{17}$ & 197.13248 & -0.005 & \\
\hline & 197.07098 & 0.08 & 12 & $\mathrm{C}_{12} \mathrm{H}_{9} \mathrm{~N}_{2} \mathrm{O}$ & 197.07094 & 0.218 & $A B E$ \\
\hline & 197.05973 & 0.12 & 19 & $\mathrm{C}_{13} \mathrm{H}_{9} \mathrm{O}_{2}$ & 197.05971 & 0.135 & \\
\hline & 196.05185 & 0.07 & 10 & $\mathrm{C}_{13} \mathrm{H}_{8} \mathrm{O}_{2}$ & 196.05188 & -0.161 & $\mathrm{HN} / \mathrm{HO}$ \\
\hline & 196.05053 & 0.36 & 62 & $\mathrm{C}_{11} \mathrm{H}_{6} \mathrm{~N}_{3} \mathrm{O}$ & 196.05054 & -0.029 & $\mathrm{~L}$ \\
\hline
\end{tabular}




\begin{tabular}{|c|c|c|c|c|c|c|c|}
\hline & $\mathrm{m} / \mathrm{z}$ & $\begin{array}{c}\text { Relative } \\
\text { intensity \% }\end{array}$ & $S / N$ & $\begin{array}{l}\text { Elemental } \\
\text { Composition }\end{array}$ & $\begin{array}{l}\text { Theoretical } \\
\text { Mass }\end{array}$ & $\begin{array}{l}\text { Error } \\
(\mathrm{ppm})\end{array}$ & Assignments \\
\hline & 191.07031 & 0.07 & 10 & $\mathrm{C}_{11} \mathrm{H}_{11} \mathrm{O}_{3}$ & 191.07027 & 0.189 & $\mathrm{~J}$ \\
\hline & 183.05528 & 0.12 & 19 & $\mathrm{C}_{11} \mathrm{H}_{7} \mathrm{~N}_{2} \mathrm{O}$ & 183.05529 & -0.037 & $\mathrm{BCM}$ \\
\hline & 181.06480 & 0.09 & 13 & $\mathrm{C}_{13} \mathrm{H}_{9} \mathrm{O}$ & 181.06479 & 0.050 & \\
\hline & 176.04679 & 0.09 & 14 & $\mathrm{C}_{10} \mathrm{H}_{8} \mathrm{O}_{3}$ & 176.04680 & -0.009 & \\
\hline & 172.03930 & 0.72 & 126 & $\mathrm{C}_{10} \mathrm{H}_{6} \mathrm{NO}_{2}$ & 172.03930 & -0.032 & J'H \\
\hline & 171.05529 & 0.10 & 16 & $\mathrm{C}_{10} \mathrm{H}_{7} \mathrm{~N}_{2} \mathrm{O}$ & 171.05529 & -0.021 & EL \\
\hline & 170.02366 & 0.16 & 27 & $\mathrm{C}_{10} \mathrm{H}_{4} \mathrm{NO}_{2}$ & 170.02365 & 0.007 & J'H \\
\hline & 169.03966 & 0.07 & 11 & $\mathrm{C}_{10} \mathrm{H}_{5} \mathrm{~N}_{2} \mathrm{O}$ & 169.03964 & 0.134 & EL \\
\hline & 165.06988 & 0.08 & 13 & $\mathrm{C}_{13} \mathrm{H}_{9}$ & 165.06988 & 0.022 & \\
\hline \multirow[t]{5}{*}{$*$} & 156.04439 & 0.17 & 28 & $\mathrm{C}_{10} \mathrm{H}_{6} \mathrm{NO}$ & 156.04439 & 0.002 & $\mathrm{HL}$ \\
\hline & 153.06988 & 0.07 & 10 & $\mathrm{C}_{12} \mathrm{H}_{9}$ & 153.06988 & 0.000 & \\
\hline & 152.06205 & 0.07 & 10 & $\mathrm{C}_{12} \mathrm{H}_{8}$ & 152.06205 & -0.016 & \\
\hline & 145.02841 & 0.09 & 14 & $\mathrm{C}_{9} \mathrm{H}_{5} \mathrm{O}_{2}$ & 145.02841 & 0.062 & \\
\hline & \multirow[t]{3}{*}{134.06005} & 0.07 & 10 & $\mathrm{C}_{8} \mathrm{H}_{8} \mathrm{NO}$ & 134.06004 & 0.091 & \\
\hline & & & & & $\begin{array}{l}\text { Standard } \\
\text { Deviation }\end{array}$ & 0.222 & \\
\hline & & & & & $\begin{array}{l}\text { Absolute } \\
\text { Average }\end{array}$ & 0.249 & \\
\hline
\end{tabular}


Table S 3: Table showing the peak list, relative intensity, assignments, and errors (ppm) of the CAD fragmentation of Fenpropidin (Figure 1a). ${ }^{*}$ displays the peaks that were used for the internal calibration.

\begin{tabular}{|c|c|c|c|c|c|c|c|}
\hline & $\mathrm{m} / \mathrm{z}$ & $\begin{array}{c}\text { Relative } \\
\text { Intensity \% }\end{array}$ & $S / N$ & $\begin{array}{l}\text { Elemental } \\
\text { Composition }\end{array}$ & $\begin{array}{l}\text { Theoretical } \\
\text { Mass }\end{array}$ & $\begin{array}{l}\text { Error } \\
(\mathrm{ppm})\end{array}$ & Assignments \\
\hline & 274.25296 & 100.00 & 11538 & $\mathrm{C}_{19} \mathrm{H}_{32} \mathrm{~N}$ & 274.25291 & 0.170 & {$[\mathrm{M}+\mathrm{H}]^{+}$} \\
\hline & 272.23733 & 0.12 & 12 & $\mathrm{C}_{19} \mathrm{H}_{30} \mathrm{~N}$ & 272.23728 & 0.184 & \\
\hline$*$ & 259.22945 & 0.27 & 30 & $\mathrm{C}_{18} \mathrm{H}_{29} \mathrm{~N}$ & 259.22945 & -0.002 & $A$ \\
\hline \multirow[t]{2}{*}{$*$} & 218.19033 & 2.36 & 282 & $\mathrm{C}_{15} \mathrm{H}_{24} \mathrm{~N}$ & 218.19033 & 0.003 & B \\
\hline & 189.16378 & 1.48 & 179 & $\mathrm{C}_{14} \mathrm{H}_{21}$ & 189.16378 & 0.035 & C \\
\hline \multirow[t]{8}{*}{$*$} & 161.13248 & 1.59 & 196 & $\mathrm{C}_{12} \mathrm{H}_{17}$ & 161.13248 & -0.001 & $\mathrm{AAC} / \mathrm{AD}$ \\
\hline & 147.11682 & 32.52 & 4041 & $\mathrm{C}_{11} \mathrm{H}_{15}$ & 147.11683 & -0.018 & $E$ \\
\hline & 145.10118 & 0.35 & 41 & $\mathrm{C}_{11} \mathrm{H}_{13}$ & 145.10118 & -0.008 & AAD \\
\hline & 133.10116 & 0.13 & 14 & $\mathrm{C}_{10} \mathrm{H}_{13}$ & 133.10118 & -0.115 & $\mathrm{~F}$ \\
\hline & 132.09335 & 0.42 & 50 & $\mathrm{C}_{10} \mathrm{H}_{12}$ & 132.09335 & -0.014 & $B C$ \\
\hline & 119.08552 & 0.99 & 122 & $\mathrm{C}_{9} \mathrm{H}_{11}$ & 119.08553 & -0.022 & $\mathrm{AF}$ \\
\hline & 117.06988 & 0.24 & 28 & $\mathrm{C}_{9} \mathrm{H}_{9}$ & 117.06988 & -0.066 & $\mathrm{BD}$ \\
\hline & 105.06987 & 0.38 & 45 & $\mathrm{C}_{8} \mathrm{H}_{9}$ & 105.06988 & -0.041 & AAF \\
\hline \multirow[t]{3}{*}{$*$} & 86.09643 & 0.16 & 18 & $\mathrm{C}_{5} \mathrm{H}_{12} \mathrm{~N}$ & 86.09643 & 0.000 & $C^{\prime}$ \\
\hline & & & & & $\begin{array}{l}\text { Standard } \\
\text { Deviation }\end{array}$ & 0.068 & \\
\hline & & & & & $\begin{array}{l}\text { Absolute } \\
\text { Average }\end{array}$ & 0.039 & \\
\hline
\end{tabular}


Table S 4: Table showing the peak list, relative intensity, assignments, and errors ( $\mathrm{ppm}$ ) of the EID MS/MS of Fenpropidin (Figure 1b). ${ }^{*}$ displays the peaks that were used for the internal calibration.

\begin{tabular}{|c|c|c|c|c|c|c|c|}
\hline & $\mathrm{m} / \mathrm{z}$ & $\begin{array}{c}\text { Relative } \\
\text { Intensity \% }\end{array}$ & $S / N$ & $\begin{array}{l}\text { Elemental } \\
\text { Composition }\end{array}$ & $\begin{array}{c}\text { Theoretical } \\
\text { Mass }\end{array}$ & $\begin{array}{l}\text { Error } \\
\text { (ppm) }\end{array}$ & Assignment \\
\hline & 274.25276 & 100.00 & 102405 & $\mathrm{C}_{19} \mathrm{H}_{32} \mathrm{~N}$ & 274.25293 & -0.623 & {$[\mathrm{M}+\mathrm{H}]^{+}$} \\
\hline & 272.23723 & 0.23 & 235 & $\mathrm{C}_{19} \mathrm{H}_{30} \mathrm{~N}$ & 272.23728 & -0.176 & \\
\hline & 260.24984 & 0.15 & 150 & $\mathrm{C}_{19} \mathrm{H}_{32}$ & 260.24985 & -0.051 & \\
\hline & 258.23420 & 0.04 & 35 & $\mathrm{C}_{19} \mathrm{H}_{30}$ & 258.23420 & -0.016 & \\
\hline * & 258.22162 & 0.20 & 205 & $\mathrm{C}_{18} \mathrm{H}_{28} \mathrm{~N}$ & 258.22163 & -0.017 & A \\
\hline & 254.20292 & 0.06 & 64 & $\mathrm{C}_{19} \mathrm{H}_{26}$ & 254.20290 & 0.074 & \\
\hline & 246.23421 & 0.17 & 174 & $\mathrm{C}_{18} \mathrm{H}_{30}$ & 246.23420 & 0.045 & \\
\hline & 244.21858 & 0.01 & 13 & $\mathrm{C}_{18} \mathrm{H}_{28}$ & 244.21855 & 0.099 & \\
\hline & 243.21073 & 0.04 & 38 & $\mathrm{C}_{18} \mathrm{H}_{27}$ & 243.21073 & 0.006 & \\
\hline & 241.19509 & 0.06 & 65 & $\mathrm{C}_{18} \mathrm{H}_{25}$ & 241.19508 & 0.053 & \\
\hline & 239.17944 & 0.10 & 108 & $\mathrm{C}_{18} \mathrm{H}_{23}$ & 239.17943 & 0.064 & \\
\hline & 232.21858 & 0.15 & 159 & $\mathrm{C}_{17} \mathrm{H}_{28}$ & 232.21855 & 0.116 & \\
\hline \multirow[t]{28}{*}{$*$} & 231.19816 & 0.02 & 15 & $\mathrm{C}_{16} \mathrm{H}_{25} \mathrm{~N}$ & 231.19815 & 0.020 & AAA \\
\hline & 225.16379 & 0.01 & 11 & $\mathrm{C}_{17} \mathrm{H}_{21}$ & 225.16378 & 0.076 & \\
\hline & 218.20293 & 0.04 & 41 & $\mathrm{C}_{16} \mathrm{H}_{26}$ & 218.20290 & 0.143 & \\
\hline & 218.19037 & 0.11 & 120 & $\mathrm{C}_{15} \mathrm{H}_{24} \mathrm{~N}$ & 218.19033 & 0.206 & B \\
\hline & 217.19512 & 0.04 & 40 & $\mathrm{C}_{16} \mathrm{H}_{25}$ & 217.19508 & 0.197 & \\
\hline & 217.18251 & 0.03 & 26 & $\mathrm{C}_{15} \mathrm{H}_{23} \mathrm{~N}$ & 217.18250 & 0.049 & \\
\hline & 216.17475 & 0.02 & 16 & $\mathrm{C}_{15} \mathrm{H}_{22} \mathrm{~N}$ & 216.17468 & 0.334 & B \\
\hline & 213.16381 & 0.02 & 17 & $\mathrm{C}_{16} \mathrm{H}_{21}$ & 213.16378 & 0.158 & \\
\hline & 211.14817 & 0.04 & 45 & $\mathrm{C}_{16} \mathrm{H}_{19}$ & 211.14813 & 0.214 & \\
\hline & 210.23427 & 0.01 & 13 & $\mathrm{C}_{15} \mathrm{H}_{30}$ & 210.23420 & 0.325 & \\
\hline & 208.21859 & 0.01 & 14 & $\mathrm{C}_{15} \mathrm{H}_{28}$ & 208.21855 & 0.172 & \\
\hline & 204.18727 & 0.02 & 18 & $\mathrm{C}_{15} \mathrm{H}_{24}$ & 204.18725 & 0.086 & \\
\hline & 203.17945 & 0.05 & 49 & $\mathrm{C}_{15} \mathrm{H}_{23}$ & 203.17943 & 0.131 & \\
\hline & 201.16377 & 0.01 & 12 & $\mathrm{C}_{15} \mathrm{H}_{21}$ & 201.16378 & -0.032 & \\
\hline & 199.14818 & 0.01 & 14 & $\mathrm{C}_{15} \mathrm{H}_{19}$ & 199.14813 & 0.253 & \\
\hline & 197.13251 & 0.06 & 62 & $\mathrm{C}_{15} \mathrm{H}_{17}$ & 197.13248 & 0.169 & \\
\hline & 196.21861 & 0.01 & 13 & $\mathrm{C}_{14} \mathrm{H}_{28}$ & 196.21855 & 0.292 & \\
\hline & 194.20290 & 0.01 & 12 & $\mathrm{C}_{14} \mathrm{H}_{26}$ & 194.20290 & -0.015 & \\
\hline & 190.17165 & 0.03 & 28 & $\mathrm{C}_{14} \mathrm{H}_{22}$ & 190.17160 & 0.250 & \\
\hline & 189.16383 & 0.06 & 66 & $\mathrm{C}_{14} \mathrm{H}_{21}$ & 189.16378 & 0.262 & C \\
\hline & 188.15600 & 0.01 & 14 & $\mathrm{C}_{14} \mathrm{H}_{20}$ & 188.15595 & 0.232 & \\
\hline & 187.14816 & 0.02 & 24 & $\mathrm{C}_{14} \mathrm{H}_{19}$ & 187.14813 & 0.153 & C \\
\hline & 185.13252 & 0.01 & 15 & $\mathrm{C}_{14} \mathrm{H}_{17}$ & 185.13248 & 0.233 & \\
\hline & 182.20285 & 0.02 & 17 & $\mathrm{C}_{13} \mathrm{H}_{26}$ & 182.20290 & -0.279 & \\
\hline & 180.18727 & 0.01 & 12 & $\mathrm{C}_{13} \mathrm{H}_{24}$ & 180.18725 & 0.101 & \\
\hline & 178.07776 & 0.02 & 15 & $\mathrm{C}_{14} \mathrm{H}_{10}$ & 178.07770 & 0.344 & \\
\hline & 176.15599 & 0.02 & 21 & $\mathrm{C}_{13} \mathrm{H}_{20}$ & 176.15595 & 0.231 & D \\
\hline & 175.14817 & 0.06 & 60 & $\mathrm{C}_{13} \mathrm{H}_{19}$ & 175.14813 & 0.257 & \\
\hline
\end{tabular}




\begin{tabular}{|c|c|c|c|c|c|c|c|}
\hline & $\mathrm{m} / \mathrm{z}$ & $\begin{array}{c}\text { Relative } \\
\text { Intensity \% }\end{array}$ & $S / N$ & $\begin{array}{l}\text { Elemental } \\
\text { Composition }\end{array}$ & $\begin{array}{c}\text { Theoretical } \\
\text { Mass }\end{array}$ & $\begin{array}{l}\text { Error } \\
(\mathrm{ppm})\end{array}$ & Assignment \\
\hline & 174.14034 & 0.01 & 12 & $\mathrm{C}_{13} \mathrm{H}_{18}$ & 174.14030 & 0.242 & D \\
\hline & 173.13253 & 0.06 & 60 & $\mathrm{C}_{13} \mathrm{H}_{17}$ & 173.13248 & 0.304 & \\
\hline & 171.11689 & 0.01 & 12 & $\mathrm{C}_{13} \mathrm{H}_{15}$ & 171.11683 & 0.369 & \\
\hline & 169.10120 & 0.01 & 11 & $\mathrm{C}_{13} \mathrm{H}_{13}$ & 169.10118 & 0.162 & \\
\hline & 168.18722 & 0.02 & 19 & $\mathrm{C}_{12} \mathrm{H}_{24}$ & 168.18725 & -0.183 & \\
\hline & 167.08560 & 0.01 & 11 & $\mathrm{C}_{13} \mathrm{H}_{11}$ & 167.08553 & 0.435 & \\
\hline & 166.17165 & 0.02 & 17 & $\mathrm{C}_{12} \mathrm{H}_{22}$ & 166.17160 & 0.317 & \\
\hline & 165.06989 & 0.02 & 26 & $\mathrm{C}_{13} \mathrm{H}_{9}$ & 165.06988 & 0.077 & \\
\hline & 163.14819 & 0.01 & 12 & $\mathrm{C}_{12} \mathrm{H}_{19}$ & 163.14813 & 0.402 & \\
\hline & 162.14032 & 0.02 & 19 & $\mathrm{C}_{12} \mathrm{H}_{18}$ & 162.14030 & 0.102 & \\
\hline & 161.13249 & 0.06 & 66 & $\mathrm{C}_{12} \mathrm{H}_{17}$ & 161.13248 & 0.075 & $A D$ \\
\hline & 159.11686 & 0.06 & 62 & $\mathrm{C}_{12} \mathrm{H}_{15}$ & 159.11683 & 0.213 & AAC \\
\hline & 155.08556 & 0.02 & 15 & $\mathrm{C}_{12} \mathrm{H}_{11}$ & 155.08553 & 0.192 & \\
\hline & 154.17163 & 0.02 & 20 & $\mathrm{C}_{11} \mathrm{H}_{22}$ & 154.17160 & 0.204 & \\
\hline & 152.06209 & 0.01 & 14 & $\mathrm{C}_{12} \mathrm{H}_{8}$ & 152.06205 & 0.276 & \\
\hline & 149.13249 & 0.01 & 14 & $\mathrm{C}_{11} \mathrm{H}_{17}$ & 149.13248 & 0.116 & \\
\hline & 148.12467 & 0.01 & 13 & $\mathrm{C}_{11} \mathrm{H}_{16}$ & 148.12465 & 0.153 & \\
\hline & 147.11684 & 0.36 & 405 & $\mathrm{C}_{11} \mathrm{H}_{15}$ & 147.11683 & 0.115 & $E$ \\
\hline & 145.10119 & 0.05 & 53 & $\mathrm{C}_{11} \mathrm{H}_{13}$ & 145.10118 & 0.122 & AAD \\
\hline & 141.06989 & 0.01 & 14 & $\mathrm{C}_{11} \mathrm{H}_{9}$ & 141.06988 & 0.092 & \\
\hline & 140.15597 & 0.02 & 18 & $\mathrm{C}_{10} \mathrm{H}_{20}$ & 140.15595 & 0.120 & \\
\hline & 138.14030 & 0.02 & 16 & $\mathrm{C}_{10} \mathrm{H}_{18}$ & 138.14030 & -0.036 & \\
\hline$*$ & 138.12773 & 0.02 & 20 & $\mathrm{C}_{9} \mathrm{H}_{16} \mathrm{~N}$ & 138.12773 & -0.004 & $F^{\prime}$ \\
\hline & 134.10900 & 0.01 & 14 & $\mathrm{C}_{10} \mathrm{H}_{14}$ & 134.10900 & -0.041 & \\
\hline & 133.10118 & 0.06 & 62 & $\mathrm{C}_{10} \mathrm{H}_{13}$ & 133.10118 & -0.002 & $\mathrm{~F}$ \\
\hline & 132.09335 & 0.06 & 65 & $\mathrm{C}_{10} \mathrm{H}_{12}$ & 132.09335 & 0.011 & \\
\hline & 131.08553 & 0.05 & 57 & $\mathrm{C}_{10} \mathrm{H}_{11}$ & 131.08553 & 0.006 & $B C$ \\
\hline & 129.06991 & 0.01 & 14 & $\mathrm{C}_{10} \mathrm{H}_{9}$ & 129.06988 & 0.241 & $\mathrm{BC} / \mathrm{AAAD}$ \\
\hline & 128.06205 & 0.01 & 14 & $\mathrm{C}_{10} \mathrm{H}_{8}$ & 128.06205 & -0.020 & \\
\hline & 126.14031 & 0.01 & 14 & $\mathrm{C}_{9} \mathrm{H}_{18}$ & 126.14030 & 0.038 & \\
\hline & 126.12773 & 0.06 & 70 & $\mathrm{C}_{8} \mathrm{H}_{16} \mathrm{~N}$ & 126.12773 & 0.003 & $E^{\prime}$ \\
\hline & 125.13248 & 0.02 & 18 & $\mathrm{C}_{9} \mathrm{H}_{17}$ & 125.13248 & 0.028 & \\
\hline & 124.12465 & 0.01 & 13 & $\mathrm{C}_{9} \mathrm{H}_{16}$ & 124.12465 & -0.055 & \\
\hline & 119.08553 & 0.09 & 100 & $\mathrm{C}_{9} \mathrm{H}_{11}$ & 119.08553 & 0.022 & $\mathrm{AF}$ \\
\hline & 117.06988 & 0.07 & 73 & $\mathrm{C}_{9} \mathrm{H}_{9}$ & 117.06988 & 0.032 & $B D$ \\
\hline & 115.05424 & 0.02 & 21 & $\mathrm{C}_{9} \mathrm{H}_{7}$ & 115.05423 & 0.086 & AF/AAE \\
\hline & 111.11683 & 0.01 & 14 & $\mathrm{C}_{8} \mathrm{H}_{15}$ & 111.11683 & 0.028 & \\
\hline$*$ & 105.06988 & 0.04 & 43 & $\mathrm{C}_{8} \mathrm{H}_{9}$ & 105.06988 & 0.001 & AAF \\
\hline & & & & & $\begin{array}{l}\text { Standard } \\
\text { Deviation }\end{array}$ & 0.112 & \\
\hline & & & & & $\begin{array}{l}\text { Absolute } \\
\text { Average }\end{array}$ & 0.140 & \\
\hline
\end{tabular}


Table S 5: Table showing the peak list, relative intensity, assignments, and errors (ppm) of the CAD fragmentation of Fluazifop acid (Figure 3a). * displays the peaks that were used for the internal calibration.

\begin{tabular}{|c|c|c|c|c|c|c|c|}
\hline & $\mathrm{m} / \mathrm{z}$ & $\begin{array}{c}\text { Relative } \\
\text { Intensity } \\
\%\end{array}$ & $S / N$ & $\begin{array}{l}\text { Elemental } \\
\text { Composition }\end{array}$ & $\begin{array}{c}\text { Theoretical } \\
\text { Mass }\end{array}$ & $\begin{array}{l}\text { Error } \\
\text { (ppm) }\end{array}$ & Assignment \\
\hline & 328.07881 & 5.36 & 915 & $\mathrm{C}_{15} \mathrm{H}_{13} \mathrm{~F}_{3} \mathrm{NO}_{4}$ & 328.07912 & -0.956 & {$[\mathrm{M}+\mathrm{H}]^{+}$} \\
\hline \multirow[t]{9}{*}{$*$} & 310.06854 & 0.09 & 13 & $\mathrm{C}_{15} \mathrm{H}_{11} \mathrm{~F}_{3} \mathrm{NO}_{3}$ & 310.06855 & -0.057 & $\mathrm{~J}$ \\
\hline & 282.07375 & 100.00 & 17700 & $\mathrm{C}_{14} \mathrm{H}_{11} \mathrm{~F}_{3} \mathrm{NO}_{2}$ & 282.07364 & 0.401 & B \\
\hline & 267.05029 & 0.17 & 29 & $\mathrm{C}_{13} \mathrm{H}_{8} \mathrm{~F}_{3} \mathrm{NO}_{2}$ & 267.05016 & 0.453 & $\mathrm{BC}$ \\
\hline & 264.06321 & 1.19 & 212 & $\mathrm{C}_{14} \mathrm{H}_{9} \mathrm{~F}_{3} \mathrm{NO}$ & 264.06308 & 0.514 & Rearrangement \\
\hline & 256.05811 & 7.63 & 1367 & $\mathrm{C}_{12} \mathrm{H}_{9} \mathrm{~F}_{3} \mathrm{NO}_{2}$ & 256.05799 & 0.472 & D \\
\hline & 255.05028 & 6.12 & 1103 & $\mathrm{C}_{12} \mathrm{H}_{8} \mathrm{~F}_{3} \mathrm{NO}_{2}$ & 255.05016 & 0.453 & \\
\hline & 254.07884 & 21.02 & 3794 & $\mathrm{C}_{13} \mathrm{H}_{11} \mathrm{~F}_{3} \mathrm{NO}$ & 254.07873 & 0.447 & Rearrangement \\
\hline & 254.04245 & 5.63 & 1014 & $\mathrm{C}_{12} \mathrm{H}_{7} \mathrm{~F}_{3} \mathrm{NO}_{2}$ & 254.04234 & 0.445 & $D$ \\
\hline & 238.04745 & 6.25 & 1140 & $\mathrm{C}_{12} \mathrm{H}_{7} \mathrm{~F}_{3} \mathrm{NO}$ & 238.04742 & 0.125 & $E$ \\
\hline \multirow[t]{4}{*}{$*$} & 236.06818 & 0.58 & 103 & $\mathrm{C}_{13} \mathrm{H}_{9} \mathrm{~F}_{3} \mathrm{~N}$ & 236.06816 & 0.084 & Rearrangement \\
\hline & 227.05519 & 0.78 & 141 & $\mathrm{C}_{11} \mathrm{H}_{8} \mathrm{~F}_{3} \mathrm{NO}$ & 227.05525 & -0.286 & $\mathrm{H}$ \\
\hline & 226.08374 & 0.45 & 80 & $\mathrm{C}_{12} \mathrm{H}_{11} \mathrm{~F}_{3} \mathrm{~N}$ & 226.08381 & -0.327 & Rearrangement \\
\hline & 214.04721 & 0.54 & 98 & $\mathrm{C}_{10} \mathrm{H}_{7} \mathrm{~F}_{3} \mathrm{NO}$ & 214.04742 & -1.007 & $\mathrm{~K}$ \\
\hline$*$ & 164.03063 & 0.75 & 143 & $\mathrm{C}_{9} \mathrm{H}_{4} \mathrm{~F}_{2} \mathrm{~N}$ & 164.03063 & -0.038 & Rearrangement \\
\hline \multirow[t]{2}{*}{$*$} & 121.02596 & 0.63 & 119 & $\mathrm{C}_{5} \mathrm{H}_{4} \mathrm{~F}_{3}$ & 121.02596 & 0.007 & 1 \\
\hline & 94.02118 & 0.51 & 97 & $\mathrm{C}_{6} \mathrm{H}_{3} \mathrm{~F}$ & 94.02133 & -1.600 & \\
\hline & & & & & $\begin{array}{l}\text { Standard } \\
\text { Deviation }\end{array}$ & 0.591 & \\
\hline & & & & & $\begin{array}{l}\text { Absolute } \\
\text { Average }\end{array}$ & 0.420 & \\
\hline
\end{tabular}


Table S 6: Table showing the peak list, relative intensity, assignments, and errors (ppm) of the EID fragmentation of Fluazifop acid (Figure $3 b$ ). ${ }^{*}$ displays the peaks that were used for the internal calibration.

\begin{tabular}{|c|c|c|c|c|c|c|c|}
\hline & $\mathrm{m} / \mathrm{z}$ & $\begin{array}{c}\text { Relative } \\
\text { Intensity \% }\end{array}$ & $S / N$ & $\begin{array}{l}\text { Elemental } \\
\text { Composition }\end{array}$ & $\begin{array}{l}\text { Theoretical } \\
\text { Mass }\end{array}$ & $\begin{array}{l}\text { Error } \\
(\mathrm{ppm})\end{array}$ & Assignment \\
\hline & 328.07914 & 100.00 & 10257 & $\mathrm{C}_{15} \mathrm{H}_{13} \mathrm{~F}_{3} \mathrm{NO}_{4}$ & 328.07912 & 0.059 & {$[\mathrm{M}+\mathrm{H}]^{+}$} \\
\hline & 327.07141 & 0.11 & 9 & $\mathrm{C}_{15} \mathrm{H}_{12} \mathrm{~F}_{3} \mathrm{NO}_{4}$ & 327.07129 & 0.367 & \\
\hline \multirow[t]{7}{*}{ * } & 282.07364 & 3.63 & 378 & $\mathrm{C}_{14} \mathrm{H}_{11} \mathrm{~F}_{3} \mathrm{NO}_{2}$ & 282.07364 & -0.007 & B \\
\hline & 268.05800 & 0.96 & 99 & $\mathrm{C}_{13} \mathrm{H}_{9} \mathrm{~F}_{3} \mathrm{NO}_{2}$ & 268.05799 & 0.039 & $B C$ \\
\hline & 256.05797 & 0.46 & 47 & $\mathrm{C}_{12} \mathrm{H}_{9} \mathrm{~F}_{3} \mathrm{NO}_{2}$ & 256.05799 & -0.063 & D \\
\hline & 255.05015 & 0.36 & 36 & $\mathrm{C}_{12} \mathrm{H}_{8} \mathrm{~F}_{3} \mathrm{NO}_{2}$ & 255.05016 & -0.042 & \\
\hline & 254.07872 & 0.41 & 41 & $\mathrm{C}_{13} \mathrm{H}_{11} \mathrm{~F}_{3} \mathrm{NO}$ & 254.07873 & -0.020 & Rearrangement \\
\hline & 254.04233 & 1.12 & 117 & $\mathrm{C}_{12} \mathrm{H}_{7} \mathrm{~F}_{3} \mathrm{NO}_{2}$ & 254.04234 & -0.049 & D \\
\hline & 240.06303 & 0.11 & 10 & $\mathrm{C}_{12} \mathrm{H}_{9} \mathrm{~F}_{3} \mathrm{NO}$ & 240.06308 & -0.203 & $E$ \\
\hline \multirow[t]{7}{*}{$*$} & 238.04743 & 0.88 & 93 & $\mathrm{C}_{12} \mathrm{H}_{7} \mathrm{~F}_{3} \mathrm{NO}$ & 238.04742 & 0.011 & $\mathrm{E}$ \\
\hline & 227.05527 & 0.26 & 27 & $\mathrm{C}_{11} \mathrm{H}_{8} \mathrm{~F}_{3} \mathrm{NO}$ & 227.05525 & 0.079 & $\mathrm{H}$ \\
\hline & 226.04745 & 0.15 & 15 & $\mathrm{C}_{11} \mathrm{H}_{7} \mathrm{~F}_{3} \mathrm{NO}$ & 226.04742 & 0.112 & \\
\hline & 218.04121 & 0.18 & 18 & $\mathrm{C}_{12} \mathrm{H}_{6} \mathrm{~F}_{2} \mathrm{NO}$ & 218.04120 & 0.074 & EM \\
\hline & 213.07840 & 0.12 & 12 & $\mathrm{C}_{13} \mathrm{H}_{11} \mathrm{NO}_{2}$ & 213.07843 & -0.159 & $B L$ \\
\hline & 198.05251 & 0.17 & 17 & $\mathrm{C}_{10} \mathrm{H}_{7} \mathrm{~F}_{3} \mathrm{~N}$ & 198.05251 & 0.017 & Rearrangement \\
\hline & 197.05971 & 0.16 & 16 & $\mathrm{C}_{13} \mathrm{H}_{9} \mathrm{O}_{2}$ & 197.05971 & 0.001 & \\
\hline \multirow[t]{5}{*}{$*$} & 182.05736 & 1.16 & 129 & $\mathrm{C}_{9} \mathrm{H}_{10} \mathrm{O}_{4}$ & 182.05736 & -0.004 & $\mathrm{G}^{\prime}$ \\
\hline & 181.06481 & 0.13 & 13 & $\mathrm{C}_{13} \mathrm{H}_{9} \mathrm{O}$ & 181.06479 & 0.117 & \\
\hline & 164.03179 & 0.14 & 14 & $\mathrm{C}_{6} \mathrm{H}_{5} \mathrm{~F}_{3} \mathrm{NO}$ & 164.03177 & 0.110 & $\mathrm{~F}$ \\
\hline & 147.02904 & 0.16 & 17 & $\mathrm{C}_{6} \mathrm{H}_{4} \mathrm{~F}_{3} \mathrm{~N}$ & 147.02904 & 0.032 & G \\
\hline & 133.10117 & 0.12 & 12 & $\mathrm{C}_{10} \mathrm{H}_{13}$ & 133.10118 & -0.071 & \\
\hline \multirow[t]{3}{*}{$*$} & 121.02841 & 0.12 & 12 & $\mathrm{C}_{7} \mathrm{H}_{5} \mathrm{O}_{2}$ & 121.02841 & 0.000 & $\mathrm{BCG}^{\prime}$ \\
\hline & & & & & $\begin{array}{l}\text { Standard } \\
\text { Deviation }\end{array}$ & 0.115 & \\
\hline & & & & & $\begin{array}{l}\text { Absolute } \\
\text { Average }\end{array}$ & 0.075 & \\
\hline
\end{tabular}


Table S 7: Table showing the peak list, relative intensity, assignments, and errors ( $p p m$ ) of the CAD fragmentation of Fluazifop-p-butyl (Figure 4a). * displays the peaks that were used for the internal calibration.

\begin{tabular}{|c|c|c|c|c|c|c|c|}
\hline & $\mathrm{m} / \mathrm{z}$ & $\begin{array}{c}\text { Relative } \\
\text { Intensity \% }\end{array}$ & $S / N$ & $\begin{array}{l}\text { Elemental } \\
\text { Composition }\end{array}$ & $\begin{array}{c}\text { Theoretical } \\
\text { Mass }\end{array}$ & $\begin{array}{l}\text { Error } \\
\text { (ppm) }\end{array}$ & Assignments \\
\hline & 384.14178 & 6.37 & 1319 & $\mathrm{C}_{19} \mathrm{H}_{21} \mathrm{~F}_{3} \mathrm{NO}_{4}$ & 384.14172 & 0.148 & {$[\mathrm{M}+\mathrm{H}]^{+}$} \\
\hline \multirow[t]{2}{*}{$*$} & 328.07912 & 44.57 & 9519 & $\mathrm{C}_{15} \mathrm{H}_{13} \mathrm{~F}_{3} \mathrm{NO}_{4}$ & 328.07912 & 0.005 & $A$ \\
\hline & 310.06858 & 0.62 & 131 & $\mathrm{C}_{15} \mathrm{H}_{11} \mathrm{~F}_{3} \mathrm{NO}_{3}$ & 310.06855 & 0.098 & $J$ \\
\hline \multirow[t]{2}{*}{$*$} & 282.07363 & 100.00 & 22115 & $\mathrm{C}_{14} \mathrm{H}_{11} \mathrm{~F}_{3} \mathrm{NO}_{2}$ & 282.07364 & -0.018 & $B$ \\
\hline & 256.05800 & 0.90 & 199 & $\mathrm{C}_{12} \mathrm{H}_{9} \mathrm{~F}_{3} \mathrm{NO}_{2}$ & 256.05799 & 0.047 & $\mathrm{D}$ \\
\hline$*$ & 254.07873 & 2.89 & 650 & $\mathrm{C}_{13} \mathrm{H}_{11} \mathrm{~F}_{3} \mathrm{NO}$ & 254.07873 & 0.022 & Rearrangement \\
\hline \multirow[t]{3}{*}{$*$} & 238.04742 & 0.17 & 37 & $\mathrm{C}_{12} \mathrm{H}_{7} \mathrm{~F}_{3} \mathrm{NO}$ & 238.04742 & -0.010 & $E$ \\
\hline & & & & & $\begin{array}{l}\text { Standard } \\
\text { Deviation }\end{array}$ & 0.043 & \\
\hline & & & & & $\begin{array}{l}\text { Absolute } \\
\text { Average }\end{array}$ & 0.033 & \\
\hline
\end{tabular}


Table S 8: Table showing the peak list, relative intensity, assignments, and errors ( $\mathrm{ppm}$ ) of the EID fragmentation of Fluazifop-p-butyl (Figure $4 b$ ). ${ }^{*}$ displays the peaks that were used for the internal calibration.

\begin{tabular}{|c|c|c|c|c|c|c|c|}
\hline & $\mathrm{m} / \mathrm{z}$ & $\begin{array}{c}\text { Relative } \\
\text { Intensity } \\
\%\end{array}$ & $S / N$ & $\begin{array}{l}\text { Elemental } \\
\text { Composition }\end{array}$ & $\begin{array}{l}\text { Theoretical } \\
\text { Mass }\end{array}$ & $\begin{array}{l}\text { Error } \\
\text { (ppm) }\end{array}$ & Assignment \\
\hline & 384.14161 & 100.00 & 25879 & $\mathrm{C}_{19} \mathrm{H}_{21} \mathrm{~F}_{3} \mathrm{NO}_{4}$ & 384.14172 & -0.297 & {$[\mathrm{M}+\mathrm{H}]^{+}$} \\
\hline & 383.13391 & 0.53 & 134 & $\mathrm{C}_{19} \mathrm{H}_{20} \mathrm{~F}_{3} \mathrm{NO}_{4}$ & 383.13389 & 0.038 & \\
\hline \multirow[t]{7}{*}{$*$} & 328.07912 & 1.89 & 505 & $\mathrm{C}_{15} \mathrm{H}_{13} \mathrm{~F}_{3} \mathrm{NO}_{4}$ & 328.07912 & 0.002 & A \\
\hline & 312.13560 & 5.09 & 1379 & $\mathrm{C}_{19} \mathrm{H}_{20} \mathrm{O}_{4}$ & 312.13561 & -0.043 & \\
\hline & 310.06855 & 0.04 & 10 & $\mathrm{C}_{15} \mathrm{H}_{11} \mathrm{~F}_{3} \mathrm{NO}_{3}$ & 310.06855 & -0.025 & J \\
\hline & 296.14070 & 0.28 & 74 & $\mathrm{C}_{19} \mathrm{H}_{20} \mathrm{O}_{3}$ & 296.14070 & 0.010 & \\
\hline & 282.07363 & 6.22 & 1717 & $\mathrm{C}_{14} \mathrm{H}_{11} \mathrm{~F}_{3} \mathrm{NO}_{2}$ & 282.07364 & -0.027 & B \\
\hline & 268.05801 & 4.16 & 1160 & $\mathrm{C}_{13} \mathrm{H}_{9} \mathrm{~F}_{3} \mathrm{NO}_{2}$ & 268.05799 & 0.069 & BC \\
\hline & 256.05799 & 0.31 & 87 & $\mathrm{C}_{12} \mathrm{H}_{9} \mathrm{~F}_{3} \mathrm{NO}_{2}$ & 256.05799 & -0.006 & D \\
\hline \multirow[t]{8}{*}{$*$} & 255.05016 & 0.79 & 221 & $\mathrm{C}_{12} \mathrm{H}_{8} \mathrm{~F}_{3} \mathrm{NO}_{2}$ & 255.05016 & -0.004 & \\
\hline & 254.07872 & 0.59 & 165 & $\mathrm{C}_{13} \mathrm{H}_{11} \mathrm{~F}_{3} \mathrm{NO}$ & 254.07873 & -0.009 & Rearrangement \\
\hline & 254.04234 & 1.05 & 294 & $\mathrm{C}_{12} \mathrm{H}_{7} \mathrm{~F}_{3} \mathrm{NO}_{2}$ & 254.04234 & -0.017 & D \\
\hline & 240.06308 & 0.51 & 144 & $\mathrm{C}_{12} \mathrm{H}_{9} \mathrm{~F}_{3} \mathrm{NO}$ & 240.06308 & 0.007 & $\mathrm{E}$ \\
\hline & 239.05525 & 0.16 & 43 & $\mathrm{C}_{12} \mathrm{H}_{8} \mathrm{~F}_{3} \mathrm{NO}$ & 239.05525 & 0.014 & \\
\hline & 238.11995 & 0.60 & 170 & $\mathrm{C}_{13} \mathrm{H}_{18} \mathrm{O}_{4}$ & 238.11996 & -0.026 & $\mathrm{G}^{\prime}$ \\
\hline & 238.04742 & 0.75 & 212 & $\mathrm{C}_{12} \mathrm{H}_{7} \mathrm{~F}_{3} \mathrm{NO}$ & 238.04742 & -0.005 & $\mathrm{E}$ \\
\hline & 227.05525 & 0.31 & 89 & $\mathrm{C}_{11} \mathrm{H}_{8} \mathrm{~F}_{3} \mathrm{NO}$ & 227.05525 & -0.021 & \\
\hline \multirow[t]{5}{*}{$*$} & 226.04743 & 0.17 & 47 & $\mathrm{C}_{11} \mathrm{H}_{7} \mathrm{~F}_{3} \mathrm{NO}$ & 226.04742 & 0.003 & $\mathrm{H}$ \\
\hline & 214.04737 & 0.04 & 10 & $\mathrm{C}_{10} \mathrm{H}_{7} \mathrm{~F}_{3} \mathrm{NO}$ & 214.04742 & -0.261 & K \\
\hline & 213.07842 & 0.11 & 30 & $\mathrm{C}_{13} \mathrm{H}_{11} \mathrm{NO}_{2}$ & 213.07843 & -0.039 & $B L$ \\
\hline & 164.03177 & 0.09 & 25 & $\mathrm{C}_{6} \mathrm{H}_{5} \mathrm{~F}_{3} \mathrm{NO}$ & 164.03177 & -0.007 & $\mathrm{~F}$ \\
\hline & 147.02905 & 0.14 & 39 & $\mathrm{C}_{6} \mathrm{H}_{4} \mathrm{~F}_{3} \mathrm{~N}$ & 147.02904 & 0.099 & \\
\hline \multirow[t]{3}{*}{$*$} & 146.02121 & 0.04 & 11 & $\mathrm{C}_{6} \mathrm{H}_{3} \mathrm{~F}_{3} \mathrm{~N}$ & 146.02121 & 0.000 & G \\
\hline & & & & & $\begin{array}{l}\text { Standard } \\
\text { Deviation }\end{array}$ & 0.065 & \\
\hline & & & & & $\begin{array}{l}\text { Absolute } \\
\text { Average }\end{array}$ & 0.033 & \\
\hline
\end{tabular}


Table S 9: Table showing the peak list, relative intensity, assignments, and errors (ppm) of the CAD fragmentation of Nicosulfuron (Figure S 1a). ${ }^{*}$ displays the peaks that were used for the internal calibration.

\begin{tabular}{|c|c|c|c|c|c|c|c|}
\hline & $\mathrm{m} / \mathrm{z}$ & $\begin{array}{c}\text { Relative } \\
\text { Intensity \% }\end{array}$ & $S / N$ & $\begin{array}{l}\text { Elemental } \\
\text { Composition }\end{array}$ & $\begin{array}{c}\text { Theoretical } \\
\text { Mass }\end{array}$ & $\begin{array}{l}\text { Error } \\
\text { (ppm) }\end{array}$ & Assignment \\
\hline \multirow[t]{11}{*}{$*$} & 366.05028 & 0.60 & 17 & $\mathrm{C}_{13} \mathrm{H}_{12} \mathrm{~N}_{5} \mathrm{O}_{6} \mathrm{~S}$ & 366.05028 & 0.001 & $A$ \\
\hline & 301.14087 & 1.66 & 51 & $\mathrm{C}_{14} \mathrm{H}_{17} \mathrm{~N}_{6} \mathrm{O}_{2}$ & 301.14075 & 0.398 & \\
\hline & 291.15649 & 0.35 & 10 & $\mathrm{C}_{13} \mathrm{H}_{19} \mathrm{~N}_{6} \mathrm{O}_{2}$ & 291.15640 & 0.303 & \\
\hline & 259.08246 & 5.47 & 176 & $\mathrm{C}_{12} \mathrm{H}_{11} \mathrm{~N}_{4} \mathrm{O}_{3}$ & 259.08257 & -0.415 & $\begin{array}{c}A E+B^{\prime} \text { (ipso- } \\
\text { bridge } \\
\text { contraction) }\end{array}$ \\
\hline & 256.03854 & 0.59 & 17 & $\mathrm{C}_{9} \mathrm{H}_{10} \mathrm{~N}_{3} \mathrm{O}_{4} \mathrm{~S}$ & 256.03865 & -0.438 & $\mathrm{~B}$ \\
\hline & 241.06816 & 0.79 & 24 & $\mathrm{C}_{15} \mathrm{H}_{13} \mathrm{OS}$ & 241.06816 & -0.012 & \\
\hline & 213.03278 & 43.33 & 1446 & $\mathrm{C}_{8} \mathrm{H}_{9} \mathrm{~N}_{2} \mathrm{O}_{3} \mathrm{~S}$ & 213.03284 & -0.283 & $\mathrm{D}$ \\
\hline & 199.13283 & 0.90 & 28 & $\mathrm{C}_{11} \mathrm{H}_{19} \mathrm{O}_{3}$ & 199.13287 & -0.197 & \\
\hline & 182.05600 & 100.00 & 3398 & $\mathrm{C}_{7} \mathrm{H}_{8} \mathrm{~N}_{3} \mathrm{O}_{3}$ & 182.05602 & -0.102 & $C^{\prime}$ \\
\hline & 163.14812 & 0.36 & 10 & $\mathrm{C}_{12} \mathrm{H}_{19}$ & 163.14813 & -0.073 & \\
\hline & 157.06077 & 1.62 & 55 & $\mathrm{C}_{6} \mathrm{H}_{9} \mathrm{~N}_{2} \mathrm{O}_{3}$ & 157.06077 & -0.019 & \\
\hline$*$ & 156.07675 & 1.60 & 54 & $\mathrm{C}_{6} \mathrm{H}_{10} \mathrm{~N}_{3} \mathrm{O}_{2}$ & 156.07675 & -0.006 & $B^{\prime}$ \\
\hline \multirow[t]{2}{*}{$*$} & 139.05020 & 0.53 & 17 & $\mathrm{C}_{6} \mathrm{H}_{7} \mathrm{~N}_{2} \mathrm{O}_{2}$ & 139.05020 & 0.007 & $\mathrm{~F}$ \\
\hline & 134.06342 & 0.35 & 11 & $\mathrm{C}_{5} \mathrm{H}_{12} \mathrm{NOS}$ & 134.06341 & 0.035 & \\
\hline \multirow[t]{3}{*}{$*$} & 106.02874 & 0.40 & 12 & $\mathrm{C}_{6} \mathrm{H}_{4} \mathrm{NO}$ & 106.02874 & -0.001 & $A E$ \\
\hline & & & & & $\begin{array}{l}\text { Standard } \\
\text { Deviation }\end{array}$ & 0.225 & \\
\hline & & & & & $\begin{array}{l}\text { Absolute } \\
\text { Average }\end{array}$ & 0.153 & \\
\hline
\end{tabular}


Table S 10: Table showing the peak list, relative intensity, assignments, and errors ( $\mathrm{ppm}$ ) of the EID fragmentation of Nicosulfuron (Figure S $1 \mathrm{~b}$ ). ${ }^{*}$ displays the peaks that were used for the internal calibration.

\begin{tabular}{|c|c|c|c|c|c|c|c|}
\hline & $\mathrm{m} / \mathrm{z}$ & $\begin{array}{l}\text { Relative } \\
\text { Intensity \% }\end{array}$ & $S / N$ & $\begin{array}{c}\text { Elemental } \\
\text { Composition }\end{array}$ & $\begin{array}{l}\text { Theoretical } \\
\text { Mass }\end{array}$ & $\begin{array}{l}\text { Error } \\
\text { (ppm) }\end{array}$ & Assignment \\
\hline & 411.10808 & 100.00 & 19206 & $\mathrm{C}_{15} \mathrm{H}_{19} \mathrm{~N}_{6} \mathrm{O}_{6} \mathrm{~S}$ & 411.10813 & -0.119 & {$[\mathrm{M}+\mathrm{H}]^{+}$} \\
\hline \multirow[t]{3}{*}{$*$} & 366.05028 & 0.56 & 109 & $\mathrm{C}_{13} \mathrm{H}_{12} \mathrm{~N}_{5} \mathrm{O}_{6} \mathrm{~S}$ & 366.05028 & 0.001 & A \\
\hline & 281.05126 & 0.13 & 25 & $\mathrm{C}_{10} \mathrm{H}_{9} \mathrm{~N}_{4} \mathrm{O}_{6}$ & 281.05166 & -1.437 & \\
\hline & 256.03873 & 0.07 & 13 & $\mathrm{C}_{9} \mathrm{H}_{10} \mathrm{~N}_{3} \mathrm{O}_{4} \mathrm{~S}$ & 256.03865 & 0.294 & B \\
\hline \multirow[t]{5}{*}{$*$} & 230.05939 & 0.09 & 18 & $\mathrm{C}_{8} \mathrm{H}_{12} \mathrm{~N}_{3} \mathrm{O}_{3} \mathrm{~S}$ & 230.05939 & 0.000 & C \\
\hline & 213.03285 & 1.06 & 230 & $\mathrm{C}_{8} \mathrm{H}_{9} \mathrm{~N}_{2} \mathrm{O}_{3} \mathrm{~S}$ & 213.03284 & 0.071 & $\mathrm{D}$ \\
\hline & 198.07476 & 0.07 & 13 & $\mathrm{C}_{7} \mathrm{H}_{10} \mathrm{~N}_{4} \mathrm{O}_{3}$ & 198.07474 & 0.075 & $D^{\prime}$ \\
\hline & 197.13249 & 0.08 & 16 & $\mathrm{C}_{15} \mathrm{H}_{17}$ & 197.13248 & 0.081 & \\
\hline & 182.05601 & 1.32 & 292 & $\mathrm{C}_{7} \mathrm{H}_{8} \mathrm{~N}_{3} \mathrm{O}_{3}$ & 182.05602 & -0.024 & $C^{\prime}$ \\
\hline \multirow[t]{3}{*}{$*$} & 156.07675 & 0.10 & 20 & $\mathrm{C}_{6} \mathrm{H}_{10} \mathrm{~N}_{3} \mathrm{O}_{2}$ & 156.07675 & 0.000 & $B^{\prime}$ \\
\hline & 155.06891 & 0.11 & 24 & $\mathrm{C}_{6} \mathrm{H}_{9} \mathrm{~N}_{3} \mathrm{O}_{2}$ & 155.06893 & -0.113 & \\
\hline & 154.06110 & 0.09 & 19 & $\mathrm{C}_{6} \mathrm{H}_{8} \mathrm{~N}_{3} \mathrm{O}_{2}$ & 154.06110 & -0.041 & $B^{\prime}$ \\
\hline \multirow[t]{3}{*}{$*$} & 106.02874 & 0.05 & 10 & $\mathrm{C}_{6} \mathrm{H}_{4} \mathrm{NO}$ & 106.02874 & 0.000 & $\mathrm{AE}$ \\
\hline & & & & & $\begin{array}{l}\text { Standard } \\
\text { Deviation }\end{array}$ & 0.435 & \\
\hline & & & & & $\begin{array}{l}\text { Absolute } \\
\text { Average }\end{array}$ & 0.174 & \\
\hline
\end{tabular}


Table S 11: Table showing the peak list, relative intensity, assignments, and errors ( $\mathrm{ppm}$ ) of the CAD fragmentation of Pirimiphos-methyl (Figure 2a). ${ }^{*}$ displays the peaks that were used for the internal calibration.

\begin{tabular}{|c|c|c|c|c|c|c|c|}
\hline & $\mathrm{m} / \mathrm{z}$ & $\begin{array}{c}\text { Relative } \\
\text { Intensity \% }\end{array}$ & $S / N$ & $\begin{array}{l}\text { Elemental } \\
\text { Composition }\end{array}$ & $\begin{array}{c}\text { Theoretical } \\
\text { Mass }\end{array}$ & $\begin{array}{l}\text { Error } \\
(\mathrm{ppm})\end{array}$ & Assignment \\
\hline & 306.10360 & 49.56 & 1073 & $\mathrm{C}_{11} \mathrm{H}_{20} \mathrm{~N}_{3} \mathrm{O}_{3} \mathrm{PSH}$ & 306.10358 & 0.070 & {$[\mathrm{M}+\mathrm{H}]^{+}$} \\
\hline & 304.08797 & 1.34 & 27 & $\mathrm{C}_{11} \mathrm{H}_{19} \mathrm{~N}_{3} \mathrm{O}_{3} \mathrm{PS}$ & 304.08793 & 0.146 & \\
\hline \multirow[t]{11}{*}{ * } & 278.07228 & 29.94 & 658 & $\mathrm{C}_{9} \mathrm{H}_{16} \mathrm{~N}_{3} \mathrm{O}_{3} \mathrm{PSH}$ & 278.07228 & 0.007 & A \\
\hline & 274.07736 & 23.57 & 521 & $\mathrm{C}_{10} \mathrm{H}_{16} \mathrm{~N}_{3} \mathrm{O}_{2} \mathrm{PSH}$ & 274.07736 & 0.003 & B \\
\hline & 264.05664 & 4.66 & 102 & $\mathrm{C}_{8} \mathrm{H}_{15} \mathrm{~N}_{3} \mathrm{O}_{3} \mathrm{PS}$ & 264.05663 & 0.066 & $\mathrm{AE}$ \\
\hline & 250.04096 & 10.64 & 237 & $\mathrm{C}_{7} \mathrm{H}_{13} \mathrm{~N}_{3} \mathrm{O}_{3} \mathrm{PS}$ & 250.04098 & -0.043 & AA \\
\hline & 246.04605 & 28.92 & 652 & $\mathrm{C}_{8} \mathrm{H}_{13} \mathrm{~N}_{3} \mathrm{O}_{2} \mathrm{PS}$ & 246.04606 & -0.043 & $A B$ \\
\hline & 242.05109 & 0.59 & 11 & $\mathrm{C}_{9} \mathrm{H}_{13} \mathrm{~N}_{3} \mathrm{OPS}$ & 242.05115 & -0.215 & \\
\hline & 236.02531 & 5.17 & 115 & $\mathrm{C}_{6} \mathrm{H}_{11} \mathrm{~N}_{3} \mathrm{O}_{3} \mathrm{PS}$ & 236.02533 & -0.074 & AAE \\
\hline & 233.01441 & 4.93 & 110 & $\mathrm{C}_{7} \mathrm{H}_{10} \mathrm{~N}_{2} \mathrm{O}_{3} \mathrm{PS}$ & 233.01443 & -0.056 & C \\
\hline & 223.06643 & 0.62 & 12 & $\mathrm{C}_{7} \mathrm{H}_{16} \mathrm{~N}_{2} \mathrm{O}_{2} \mathrm{PS}$ & 223.06646 & -0.128 & \\
\hline & 218.01474 & 4.32 & 98 & $\mathrm{C}_{6} \mathrm{H}_{9} \mathrm{~N}_{3} \mathrm{O}_{2} \mathrm{PS}$ & 218.01476 & -0.082 & $A A B$ \\
\hline & 214.11035 & 0.53 & 10 & $\mathrm{C}_{9} \mathrm{H}_{17} \mathrm{~N}_{3} \mathrm{OP}$ & 214.11038 & -0.099 & BBD \\
\hline \multirow[t]{10}{*}{ * } & 208.01917 & 1.61 & 35 & $\mathrm{C}_{6} \mathrm{H}_{11} \mathrm{NO}_{3} \mathrm{PS}$ & 208.01918 & -0.011 & L \\
\hline & 207.08929 & 9.65 & 221 & $\mathrm{C}_{7} \mathrm{H}_{16} \mathrm{~N}_{2} \mathrm{O}_{3} \mathrm{P}$ & 207.08931 & -0.051 & DEF \\
\hline & 196.14444 & 5.94 & 137 & $\mathrm{C}_{10} \mathrm{H}_{18} \mathrm{~N}_{3} \mathrm{O}$ & 196.14444 & -0.009 & \\
\hline & 196.09030 & 1.09 & 24 & $\mathrm{C}_{9} \mathrm{H}_{14} \mathrm{~N}_{3} \mathrm{~S}$ & 196.09029 & 0.044 & Rearrangement \\
\hline & 195.08928 & 0.88 & 19 & $\mathrm{C}_{6} \mathrm{H}_{16} \mathrm{~N}_{2} \mathrm{O}_{3} \mathrm{P}$ & 195.08931 & -0.115 & \\
\hline & 191.05801 & 0.62 & 13 & $\mathrm{C}_{6} \mathrm{H}_{12} \mathrm{~N}_{2} \mathrm{O}_{3} \mathrm{P}$ & 191.05801 & 0.001 & ADF \\
\hline & 184.09030 & 0.55 & 11 & $\mathrm{C}_{8} \mathrm{H}_{14} \mathrm{~N}_{3} \mathrm{~S}$ & 184.09029 & 0.006 & Rearrangement \\
\hline & 179.05800 & 8.52 & 200 & $\mathrm{C}_{5} \mathrm{H}_{12} \mathrm{~N}_{2} \mathrm{O}_{3} \mathrm{P}$ & 179.05801 & -0.007 & ADEF \\
\hline & 168.11314 & 4.39 & 103 & $\mathrm{C}_{8} \mathrm{H}_{14} \mathrm{~N}_{3} \mathrm{O}$ & 168.11314 & -0.005 & EG \\
\hline & 164.11822 & 100.00 & 2387 & $\mathrm{C}_{9} \mathrm{H}_{14} \mathrm{~N}_{3}$ & 164.11822 & -0.001 & $\mathrm{H}$ \\
\hline \multirow[t]{6}{*}{$*$} & 151.02671 & 3.43 & 80 & $\mathrm{C}_{3} \mathrm{H}_{8} \mathrm{~N}_{2} \mathrm{O}_{3} \mathrm{P}$ & 151.02671 & 0.005 & AADEF \\
\hline & 142.99263 & 6.56 & 155 & $\mathrm{C}_{2} \mathrm{H}_{8} \mathrm{O}_{3} \mathrm{PS}$ & 142.99263 & 0.003 & $\mathrm{H}^{\prime}$ \\
\hline & 136.08692 & 9.06 & 215 & $\mathrm{C}_{7} \mathrm{H}_{10} \mathrm{~N}_{3}$ & 136.08692 & 0.004 & $\mathrm{AH}$ \\
\hline & 123.09169 & 1.04 & 23 & $\mathrm{C}_{7} \mathrm{H}_{11} \mathrm{~N}_{2}$ & 123.09167 & 0.096 & 1 \\
\hline & 109.07603 & 1.44 & 33 & $\mathrm{C}_{6} \mathrm{H}_{9} \mathrm{~N}_{2}$ & 109.07602 & 0.022 & El \\
\hline & 108.05563 & 5.10 & 120 & $\mathrm{C}_{5} \mathrm{H}_{6} \mathrm{~N}_{3}$ & 108.05562 & 0.037 & AAH \\
\hline \multirow[t]{3}{*}{$*$} & 95.06037 & 0.73 & 15 & $\mathrm{C}_{5} \mathrm{H}_{7} \mathrm{~N}_{2}$ & 95.06037 & 0.000 & $\mathrm{CH}$ \\
\hline & & & & & $\begin{array}{l}\text { Standard } \\
\text { Deviation }\end{array}$ & 0.071 & \\
\hline & & & & & $\begin{array}{l}\text { Absolute } \\
\text { Average }\end{array}$ & 0.048 & \\
\hline
\end{tabular}


Table S 12: Table showing the peak list, relative intensity, assignments, and errors (ppm) of the EID fragmentation of Pirimiphos-methyl (Figure 2b). ${ }^{*}$ displays the peaks that were used for the internal calibration.

\begin{tabular}{|c|c|c|c|c|c|c|c|}
\hline & $\mathrm{m} / \mathrm{z}$ & $\begin{array}{c}\text { Relative } \\
\text { Intensity \% }\end{array}$ & $S / N$ & $\begin{array}{l}\text { Elemental } \\
\text { Composition }\end{array}$ & $\begin{array}{l}\text { Theoretical } \\
\text { Mass }\end{array}$ & $\begin{array}{l}\text { Error } \\
(\mathrm{ppm})\end{array}$ & Assignment \\
\hline & 306.10357 & 100.00 & 115754 & $\mathrm{C}_{11} \mathrm{H}_{21} \mathrm{~N}_{3} \mathrm{O}_{3} \mathrm{PS}$ & 306.10358 & -0.026 & {$[\mathrm{M}+\mathrm{H}]^{+}$} \\
\hline & 304.08792 & 0.04 & 43 & $\mathrm{C}_{11} \mathrm{H}_{19} \mathrm{~N}_{3} \mathrm{O}_{3} \mathrm{PS}$ & 304.08793 & -0.024 & \\
\hline & 290.07228 & 0.20 & 235 & $\mathrm{C}_{10} \mathrm{H}_{17} \mathrm{~N}_{3} \mathrm{O}_{3} \mathrm{PS}$ & 290.07228 & 0.009 & $E$ \\
\hline & 287.22170 & 1.25 & 1451 & $\mathrm{C}_{16} \mathrm{H}_{31} \mathrm{O}_{4}$ & 287.22169 & 0.037 & \\
\hline \multirow[t]{16}{*}{ * } & 278.07227 & 0.96 & 1121 & $\mathrm{C}_{9} \mathrm{H}_{16} \mathrm{~N}_{3} \mathrm{O}_{3} \mathrm{PSH}$ & 278.07228 & -0.008 & A \\
\hline & 276.05662 & 0.14 & 165 & $\mathrm{C}_{9} \mathrm{H}_{15} \mathrm{~N}_{3} \mathrm{O}_{3} \mathrm{PS}$ & 276.05663 & -0.010 & A \\
\hline & 275.08522 & 0.02 & 18 & $\mathrm{C}_{10} \mathrm{H}_{18} \mathrm{~N}_{3} \mathrm{O}_{2} \mathrm{PS}$ & 275.08519 & 0.132 & \\
\hline & 274.13150 & 0.57 & 667 & $\mathrm{C}_{11} \mathrm{H}_{21} \mathrm{~N}_{3} \mathrm{O}_{3} \mathrm{P}$ & 274.13150 & -0.002 & D \\
\hline & 274.07736 & 0.87 & 1026 & $\mathrm{C}_{10} \mathrm{H}_{17} \mathrm{~N}_{3} \mathrm{O}_{2} \mathrm{PS}$ & 274.07736 & -0.005 & B \\
\hline & 272.11583 & 0.01 & 15 & $\mathrm{C}_{11} \mathrm{H}_{19} \mathrm{~N}_{3} \mathrm{O}_{3} \mathrm{P}$ & 272.11585 & -0.090 & D \\
\hline & 262.05354 & 0.05 & 53 & $\mathrm{C}_{9} \mathrm{H}_{15} \mathrm{~N}_{2} \mathrm{O}_{3} \mathrm{PS}$ & 262.05355 & -0.053 & M \\
\hline & 262.04096 & 0.05 & 53 & $\mathrm{C}_{8} \mathrm{H}_{13} \mathrm{~N}_{3} \mathrm{O}_{3} \mathrm{PS}$ & 262.04098 & -0.070 & $\mathrm{AE}$ \\
\hline & 258.10020 & 0.02 & 25 & $\mathrm{C}_{10} \mathrm{H}_{17} \mathrm{~N}_{3} \mathrm{O}_{3} \mathrm{P}$ & 258.10020 & -0.020 & $\mathrm{DE}$ \\
\hline & 250.04096 & 0.22 & 263 & $\mathrm{C}_{7} \mathrm{H}_{13} \mathrm{~N}_{3} \mathrm{O}_{3} \mathrm{PS}$ & 250.04098 & -0.042 & $A A$ \\
\hline & 247.12058 & 0.03 & 33 & $\mathrm{C}_{10} \mathrm{H}_{20} \mathrm{~N}_{2} \mathrm{O}_{3} \mathrm{P}$ & 247.12061 & -0.087 & \\
\hline & 246.10018 & 0.02 & 18 & $\mathrm{C}_{9} \mathrm{H}_{17} \mathrm{~N}_{3} \mathrm{O}_{3} \mathrm{P}$ & 246.10020 & -0.096 & $A D$ \\
\hline & 246.04605 & 0.61 & 725 & $\mathrm{C}_{8} \mathrm{H}_{13} \mathrm{~N}_{3} \mathrm{O}_{2} \mathrm{PS}$ & 246.04606 & -0.032 & $A B$ \\
\hline & 244.03040 & 0.05 & 63 & $\mathrm{C}_{8} \mathrm{H}_{11} \mathrm{~N}_{3} \mathrm{O}_{2} \mathrm{PS}$ & 244.03041 & -0.039 & $A B$ \\
\hline & 242.10529 & 0.22 & 259 & $\mathrm{C}_{10} \mathrm{H}_{17} \mathrm{~N}_{3} \mathrm{O}_{2} \mathrm{P}$ & 242.10529 & -0.015 & $\mathrm{BD}$ \\
\hline & 235.03007 & 0.02 & 22 & $\mathrm{C}_{7} \mathrm{H}_{12} \mathrm{~N}_{2} \mathrm{O}_{3} \mathrm{PS}$ & 235.03008 & -0.044 & C \\
\hline \multirow[t]{19}{*}{$*$} & 233.01443 & 0.20 & 243 & $\mathrm{C}_{7} \mathrm{H}_{10} \mathrm{~N}_{2} \mathrm{O}_{3} \mathrm{PS}$ & 233.01443 & 0.010 & C \\
\hline & 230.06892 & 0.01 & 15 & $\mathrm{C}_{8} \mathrm{H}_{13} \mathrm{~N}_{3} \mathrm{O}_{3} \mathrm{P}$ & 230.06890 & 0.089 & $\mathrm{AE}$ \\
\hline & 230.01475 & 0.01 & 13 & $\mathrm{C}_{7} \mathrm{H}_{9} \mathrm{~N}_{3} \mathrm{O}_{2} \mathrm{PS}$ & 230.01476 & -0.033 & $A B E$ \\
\hline & 228.08966 & 0.01 & 16 & $\mathrm{C}_{9} \mathrm{H}_{15} \mathrm{~N}_{3} \mathrm{O}_{2} \mathrm{P}$ & 228.08964 & 0.108 & $\mathrm{BDE}$ \\
\hline & 223.06648 & 0.03 & 31 & $\mathrm{C}_{7} \mathrm{H}_{16} \mathrm{~N}_{2} \mathrm{O}_{2} \mathrm{PS}$ & 223.06646 & 0.071 & \\
\hline & 219.08933 & 0.01 & 10 & $\mathrm{C}_{8} \mathrm{H}_{16} \mathrm{~N}_{2} \mathrm{O}_{3} \mathrm{P}$ & 219.08931 & 0.097 & DF \\
\hline & 218.01476 & 0.10 & 121 & $\mathrm{C}_{6} \mathrm{H}_{9} \mathrm{~N}_{3} \mathrm{O}_{2} \mathrm{PS}$ & 218.01476 & 0.010 & $A A B$ \\
\hline & 214.11039 & 0.03 & 32 & $\mathrm{C}_{9} \mathrm{H}_{17} \mathrm{~N}_{3} \mathrm{OP}$ & 214.11038 & 0.057 & BBD \\
\hline & 213.06615 & 0.01 & 9 & $\mathrm{C}_{8} \mathrm{H}_{12} \mathrm{~N}_{3} \mathrm{O}_{2} \mathrm{P}$ & 213.06616 & -0.087 & $A B D$ \\
\hline & 210.07907 & 0.01 & 10 & $\mathrm{C}_{9} \mathrm{H}_{13} \mathrm{~N}_{3} \mathrm{OP}$ & 210.07908 & -0.006 & BBD \\
\hline & 208.01919 & 0.02 & 28 & $\mathrm{C}_{6} \mathrm{H}_{11} \mathrm{NO}_{3} \mathrm{PS}$ & 208.01918 & 0.068 & L \\
\hline & 207.08931 & 0.31 & 385 & $\mathrm{C}_{7} \mathrm{H}_{16} \mathrm{~N}_{2} \mathrm{O}_{3} \mathrm{P}$ & 207.08931 & 0.008 & DEF \\
\hline & 203.00386 & 0.02 & 28 & $\mathrm{C}_{6} \mathrm{H}_{8} \mathrm{~N}_{2} \mathrm{O}_{2} \mathrm{PS}$ & 203.00386 & -0.030 & $B C$ \\
\hline & 199.16926 & 0.42 & 527 & $\mathrm{C}_{12} \mathrm{H}_{23} \mathrm{O}_{2}$ & 199.16926 & 0.008 & \\
\hline & 197.09812 & 0.05 & 55 & $\mathrm{C}_{9} \mathrm{H}_{15} \mathrm{~N}_{3} \mathrm{~S}$ & 197.09812 & -0.001 & \\
\hline & 196.14444 & 0.24 & 296 & $\mathrm{C}_{10} \mathrm{H}_{18} \mathrm{~N}_{3} \mathrm{O}$ & 196.14444 & -0.003 & \\
\hline & 196.09029 & 0.06 & 77 & $\mathrm{C}_{9} \mathrm{H}_{14} \mathrm{~N}_{3} \mathrm{~S}$ & 196.09029 & -0.016 & Rearrangement \\
\hline & 195.13662 & 0.04 & 48 & $\mathrm{C}_{10} \mathrm{H}_{17} \mathrm{~N}_{3} \mathrm{O}$ & 195.13661 & 0.048 & \\
\hline & 194.12880 & 0.01 & 10 & $\mathrm{C}_{10} \mathrm{H}_{16} \mathrm{~N}_{3} \mathrm{O}$ & 194.12879 & 0.042 & \\
\hline
\end{tabular}




\begin{tabular}{|c|c|c|c|c|c|c|c|}
\hline & $\mathrm{m} / \mathrm{z}$ & $\begin{array}{c}\text { Relative } \\
\text { Intensity \% }\end{array}$ & $S / N$ & $\begin{array}{l}\text { Elemental } \\
\text { Composition }\end{array}$ & $\begin{array}{l}\text { Theoretical } \\
\text { Mass }\end{array}$ & $\begin{array}{l}\text { Error } \\
(\mathrm{ppm})\end{array}$ & Assignment \\
\hline & 194.07464 & 0.01 & 12 & $\mathrm{C}_{9} \mathrm{H}_{12} \mathrm{~N}_{3} \mathrm{~S}$ & 194.07464 & -0.034 & \\
\hline & 191.98789 & 0.01 & 9 & $\mathrm{C}_{5} \mathrm{H}_{7} \mathrm{NO}_{3} \mathrm{PS}$ & 191.98788 & 0.048 & CM \\
\hline & 191.05801 & 0.01 & 11 & $\mathrm{C}_{6} \mathrm{H}_{12} \mathrm{~N}_{2} \mathrm{O}_{3} \mathrm{P}$ & 191.05801 & 0.020 & ADF \\
\hline & 182.12879 & 0.04 & 43 & $\mathrm{C}_{9} \mathrm{H}_{16} \mathrm{~N}_{3} \mathrm{O}$ & 182.12879 & -0.010 & G \\
\hline & 182.07464 & 0.05 & 62 & $\mathrm{C}_{8} \mathrm{H}_{12} \mathrm{~N}_{3} \mathrm{~S}$ & 182.07464 & -0.009 & Rearrangement \\
\hline & 181.12096 & 0.05 & 58 & $\mathrm{C}_{9} \mathrm{H}_{15} \mathrm{~N}_{3} \mathrm{O}$ & 181.12096 & -0.001 & \\
\hline & 180.11314 & 0.27 & 342 & $\mathrm{C}_{9} \mathrm{H}_{14} \mathrm{~N}_{3} \mathrm{O}$ & 180.11314 & -0.002 & G \\
\hline & 179.05800 & 0.12 & 156 & $\mathrm{C}_{5} \mathrm{H}_{12} \mathrm{~N}_{2} \mathrm{O}_{3} \mathrm{P}$ & 179.05801 & -0.003 & ADEF \\
\hline & 177.07873 & 0.03 & 33 & $\mathrm{C}_{6} \mathrm{H}_{14} \mathrm{~N}_{2} \mathrm{O}_{2} \mathrm{P}$ & 177.07874 & -0.055 & \\
\hline & 173.11723 & 0.01 & 9 & $\mathrm{C}_{9} \mathrm{H}_{17} \mathrm{O}_{3}$ & 173.11722 & 0.047 & \\
\hline \multirow[t]{25}{*}{$*$} & 168.11314 & 0.08 & 103 & $\mathrm{C}_{8} \mathrm{H}_{14} \mathrm{~N}_{3} \mathrm{O}$ & 168.11314 & -0.003 & EG \\
\hline & 168.05899 & 0.05 & 66 & $\mathrm{C}_{7} \mathrm{H}_{10} \mathrm{~N}_{3} \mathrm{~S}$ & 168.05899 & -0.001 & Rearrangement \\
\hline & 167.98788 & 0.01 & 13 & $\mathrm{C}_{3} \mathrm{H}_{7} \mathrm{NO}_{3} \mathrm{PS}$ & 167.98788 & 0.014 & $\mathrm{~J}$ \\
\hline & 166.09750 & 0.02 & 25 & $\mathrm{C}_{8} \mathrm{H}_{12} \mathrm{~N}_{3} \mathrm{O}$ & 166.09749 & 0.056 & EG \\
\hline & 166.04333 & 0.01 & 12 & $\mathrm{C}_{7} \mathrm{H}_{8} \mathrm{~N}_{3} \mathrm{~S}$ & 166.04334 & -0.119 & Rearrangement \\
\hline & 165.12605 & 0.12 & 153 & $\mathrm{C}_{9} \mathrm{H}_{15} \mathrm{~N}_{3}$ & 165.12605 & -0.024 & \\
\hline & 164.11822 & 0.79 & 1019 & $\mathrm{C}_{9} \mathrm{H}_{14} \mathrm{~N}_{3}$ & 164.11822 & -0.014 & $\mathrm{H}$ \\
\hline & 163.11040 & 0.05 & 56 & $\mathrm{C}_{9} \mathrm{H}_{13} \mathrm{~N}_{3}$ & 163.11040 & -0.020 & \\
\hline & 159.03179 & 0.01 & 10 & $\mathrm{C}_{5} \mathrm{H}_{8} \mathrm{~N}_{2} \mathrm{O}_{2} \mathrm{P}$ & 159.03179 & -0.033 & \\
\hline & 154.05594 & 0.01 & 11 & $\mathrm{C}_{7} \mathrm{H}_{10} \mathrm{~N}_{2} \mathrm{~S}$ & 154.05592 & 0.136 & Rearrangement \\
\hline & 154.04334 & 0.01 & 15 & $\mathrm{C}_{6} \mathrm{H}_{8} \mathrm{~N}_{3} \mathrm{~S}$ & 154.04334 & -0.010 & Rearrangement \\
\hline & 152.08184 & 0.07 & 88 & $\mathrm{C}_{7} \mathrm{H}_{10} \mathrm{~N}_{3} \mathrm{O}$ & 152.08184 & -0.016 & AG \\
\hline & 151.02670 & 0.07 & 20 & $\mathrm{C}_{3} \mathrm{H}_{8} \mathrm{~N}_{2} \mathrm{O}_{3} \mathrm{P}$ & 151.02671 & -0.003 & AADEF \\
\hline & 150.10257 & 0.23 & 302 & $\mathrm{C}_{8} \mathrm{H}_{12} \mathrm{~N}_{3}$ & 150.10257 & -0.001 & EH \\
\hline & 150.03146 & 0.01 & 14 & $\mathrm{C}_{4} \mathrm{H}_{9} \mathrm{NO}_{3} \mathrm{P}$ & 150.03146 & 0.008 & DI' \\
\hline & 140.08185 & 0.01 & 11 & $\mathrm{C}_{6} \mathrm{H}_{10} \mathrm{~N}_{3} \mathrm{O}$ & 140.08184 & 0.111 & AEG \\
\hline & 138.06621 & 0.01 & 15 & $\mathrm{C}_{6} \mathrm{H}_{8} \mathrm{~N}_{3} \mathrm{O}$ & 138.06619 & 0.134 & AEG \\
\hline & 136.08693 & 0.09 & 120 & $\mathrm{C}_{7} \mathrm{H}_{10} \mathrm{~N}_{3}$ & 136.08692 & 0.020 & $\mathrm{AH}$ \\
\hline & 134.96640 & 0.01 & 11 & $\mathrm{C}_{3} \mathrm{H}_{4} \mathrm{O}_{2} \mathrm{PS}$ & 134.96641 & -0.109 & BK \\
\hline & 134.07128 & 0.01 & 12 & $\mathrm{C}_{7} \mathrm{H}_{8} \mathrm{~N}_{3}$ & 134.07127 & 0.034 & $\mathrm{AH}$ \\
\hline & 133.10118 & 0.01 & 14 & $\mathrm{C}_{10} \mathrm{H}_{13}$ & 133.10118 & 0.035 & \\
\hline & 124.98206 & 0.05 & 62 & $\mathrm{C}_{2} \mathrm{H}_{6} \mathrm{O}_{2} \mathrm{PS}$ & 124.98206 & -0.061 & $\mathrm{G}^{\prime}$ \\
\hline & 123.09167 & 0.02 & 26 & $\mathrm{C}_{7} \mathrm{H}_{11} \mathrm{~N}_{2}$ & 123.09167 & -0.023 & 1 \\
\hline & 111.11683 & 0.01 & 12 & $\mathrm{C}_{8} \mathrm{H}_{15}$ & 111.11683 & 0.013 & \\
\hline & 109.07602 & 0.01 & 12 & $\mathrm{C}_{6} \mathrm{H}_{9} \mathrm{~N}_{2}$ & 109.07602 & -0.008 & El \\
\hline \multirow[t]{3}{*}{$*$} & 108.05562 & 0.02 & 27 & $\mathrm{C}_{5} \mathrm{H}_{6} \mathrm{~N}_{3}$ & 108.05562 & 0.000 & AAH \\
\hline & & & & & $\begin{array}{l}\text { Standard } \\
\text { Deviation }\end{array}$ & 0.054 & \\
\hline & & & & & $\begin{array}{l}\text { Absolute } \\
\text { Average }\end{array}$ & 0.038 & \\
\hline
\end{tabular}


Table S 13: Table showing the peak list, relative intensity, assignments, and errors ( $\mathrm{ppm}$ ) of the CAD fragmentation of s-metolachlor (Figure S 3a). * displays the peaks that were used for the internal calibration.

\begin{tabular}{|c|c|c|c|c|c|c|c|}
\hline & $\mathrm{m} / \mathrm{z}$ & $\begin{array}{l}\text { Relative } \\
\text { Intensity \% }\end{array}$ & $S / N$ & $\begin{array}{c}\text { Elemental } \\
\text { Composition }\end{array}$ & $\begin{array}{l}\text { Theoretical } \\
\text { mass }\end{array}$ & $\begin{array}{l}\text { Error } \\
\text { (ppm) }\end{array}$ & Assignment \\
\hline \multirow[t]{4}{*}{$*$} & 252.11498 & 100.00 & 1793 & $\mathrm{C}_{14} \mathrm{H}_{19} \mathrm{ClNO}$ & 252.11497 & 0.029 & A \\
\hline & 224.12006 & 1.50 & 25 & $\mathrm{C}_{13} \mathrm{H}_{19} \mathrm{ClN}$ & 224.12005 & 0.034 & \\
\hline & 224.08371 & 0.72 & 11 & $\mathrm{C}_{12} \mathrm{H}_{15} \mathrm{CINO}$ & 224.08367 & 0.181 & $B C$ \\
\hline & 213.12738 & 0.90 & 14 & $\mathrm{C}_{15} \mathrm{H}_{17} \mathrm{O}$ & 213.12739 & -0.037 & \\
\hline \multirow[t]{3}{*}{$*$} & 212.08366 & 1.99 & 35 & $\mathrm{C}_{11} \mathrm{H}_{15} \mathrm{CINO}$ & 212.08367 & -0.056 & D \\
\hline & 194.07312 & 1.26 & 21 & $\mathrm{C}_{11} \mathrm{H}_{13} \mathrm{CIN}$ & 194.07310 & 0.091 & \\
\hline & 184.05238 & 1.34 & 23 & $\mathrm{C}_{9} \mathrm{H}_{11} \mathrm{CINO}$ & 184.05237 & 0.074 & \\
\hline \multirow[t]{8}{*}{ * } & 176.14338 & 75.73 & 1422 & $\mathrm{C}_{12} \mathrm{H}_{18} \mathrm{~N}$ & 176.14338 & 0.031 & $A E$ \\
\hline & 174.12772 & 0.68 & 11 & $\mathrm{C}_{12} \mathrm{H}_{16} \mathrm{~N}$ & 174.12773 & -0.016 & $\mathrm{AE}$ \\
\hline & 160.11207 & 1.98 & 36 & $\mathrm{C}_{11} \mathrm{H}_{14} \mathrm{~N}$ & 160.11208 & -0.006 & $\mathrm{BE}$ \\
\hline & 159.10427 & 0.70 & 12 & $\mathrm{C}_{11} \mathrm{H}_{13} \mathrm{~N}$ & 159.10425 & 0.111 & \\
\hline & 158.09643 & 1.50 & 27 & $\mathrm{C}_{11} \mathrm{H}_{12} \mathrm{~N}$ & 158.09643 & 0.003 & $\mathrm{BE}$ \\
\hline & 148.11209 & 0.75 & 13 & $\mathrm{C}_{10} \mathrm{H}_{14} \mathrm{~N}$ & 148.11208 & 0.101 & BCE \\
\hline & 146.09643 & 1.65 & 30 & $\mathrm{C}_{10} \mathrm{H}_{12} \mathrm{~N}$ & 146.09643 & -0.005 & BCE \\
\hline & 145.10117 & 0.63 & 10 & $\mathrm{C}_{11} \mathrm{H}_{13}$ & 145.10118 & -0.081 & \\
\hline \multirow[t]{3}{*}{$*$} & 134.09643 & 2.84 & 54 & $\mathrm{C}_{9} \mathrm{H}_{12} \mathrm{~N}$ & 134.09643 & -0.004 & $\mathrm{DE}$ \\
\hline & & & & & $\begin{array}{l}\text { Standard } \\
\text { Deviation }\end{array}$ & 0.069 & \\
\hline & & & & & $\begin{array}{l}\text { Absolute } \\
\text { Average }\end{array}$ & 0.054 & \\
\hline
\end{tabular}


Table S 14: Table showing the peak list, relative intensity, assignments, and errors ( $\mathrm{ppm}$ ) of the EID fragmentation of $s$-metolachlor (Figure $S 3 b$ ). ${ }^{*}$ displays the peaks that were used for the internal calibration.

\begin{tabular}{|c|c|c|c|c|c|c|c|}
\hline & $\mathrm{m} / \mathrm{z}$ & $\begin{array}{c}\text { Relative } \\
\text { Intensity \% }\end{array}$ & $S / N$ & $\begin{array}{l}\text { Elemental } \\
\text { Composition }\end{array}$ & $\begin{array}{c}\text { Theoretical } \\
\text { Mass }\end{array}$ & $\begin{array}{l}\text { Error } \\
\text { (ppm) }\end{array}$ & Assignment \\
\hline & 284.14105 & 100.00 & 26359 & $\mathrm{C}_{15} \mathrm{H}_{23} \mathrm{CINO}_{2}$ & 284.14118 & -0.471 & {$[\mathrm{M}+\mathrm{H}]^{+}$} \\
\hline \multirow[t]{12}{*}{ * } & 252.11497 & 7.26 & 1952 & $\mathrm{C}_{14} \mathrm{H}_{19} \mathrm{ClNO}$ & 252.11497 & 0.000 & A \\
\hline & 249.17240 & 0.05 & 12 & $\mathrm{C}_{15} \mathrm{H}_{23} \mathrm{NO}_{2}$ & 249.17233 & 0.270 & $\mathrm{~F}$ \\
\hline & 238.09933 & 0.25 & 67 & $\mathrm{C}_{13} \mathrm{H}_{17} \mathrm{ClNO}$ & 238.09932 & 0.036 & B \\
\hline & 234.14887 & 0.17 & 44 & $\mathrm{C}_{14} \mathrm{H}_{20} \mathrm{NO}_{2}$ & 234.14886 & 0.082 & G \\
\hline & 224.12000 & 0.06 & 15 & $\mathrm{C}_{13} \mathrm{H}_{19} \mathrm{ClN}$ & 224.12005 & -0.220 & \\
\hline & 220.13321 & 0.09 & 24 & $\mathrm{C}_{13} \mathrm{H}_{18} \mathrm{NO}_{2}$ & 220.13321 & 0.026 & CG \\
\hline & 216.13827 & 0.06 & 15 & $\mathrm{C}_{14} \mathrm{H}_{18} \mathrm{NO}$ & 216.13829 & -0.104 & $\mathrm{AF}$ \\
\hline & 212.08363 & 0.07 & 19 & $\mathrm{C}_{11} \mathrm{H}_{15} \mathrm{ClNO}$ & 212.08367 & -0.189 & D \\
\hline & 211.07586 & 0.06 & 14 & $\mathrm{C}_{11} \mathrm{H}_{14} \mathrm{ClNO}$ & 211.07584 & 0.092 & \\
\hline & 204.13828 & 1.99 & 556 & $\mathrm{C}_{13} \mathrm{H}_{18} \mathrm{NO}$ & 204.13829 & -0.031 & $\mathrm{BF}$ \\
\hline & 202.12265 & 0.28 & 76 & $\mathrm{C}_{13} \mathrm{H}_{16} \mathrm{NO}$ & 202.12264 & 0.033 & $A G$ \\
\hline & 197.05970 & 0.05 & 11 & $\mathrm{C}_{13} \mathrm{H}_{9} \mathrm{O}_{2}$ & 197.05971 & -0.009 & \\
\hline \multirow[t]{11}{*}{ * } & 190.12264 & 0.13 & 35 & $\mathrm{C}_{12} \mathrm{H}_{16} \mathrm{NO}$ & 190.12264 & 0.000 & $B G$ \\
\hline & 188.10699 & 0.18 & 48 & $\mathrm{C}_{12} \mathrm{H}_{14} \mathrm{NO}$ & 188.10699 & -0.011 & BG \\
\hline & 186.12772 & 0.12 & 31 & $\mathrm{C}_{13} \mathrm{H}_{16} \mathrm{~N}$ & 186.12773 & -0.042 & \\
\hline & 184.05237 & 0.13 & 35 & $\mathrm{C}_{9} \mathrm{H}_{11} \mathrm{CINO}$ & 184.05237 & -0.007 & \\
\hline & 177.11481 & 0.15 & 42 & $\mathrm{C}_{11} \mathrm{H}_{15} \mathrm{NO}$ & 177.11482 & -0.053 & DF \\
\hline & 176.14337 & 0.69 & 196 & $\mathrm{C}_{12} \mathrm{H}_{18} \mathrm{~N}$ & 176.14338 & -0.056 & $\mathrm{AE}$ \\
\hline & 176.10699 & 0.09 & 23 & $\mathrm{C}_{11} \mathrm{H}_{14} \mathrm{NO}$ & 176.10699 & -0.023 & \\
\hline & 174.12771 & 0.06 & 15 & $\mathrm{C}_{12} \mathrm{H}_{16} \mathrm{~N}$ & 174.12773 & -0.075 & $\mathrm{AE}$ \\
\hline & 162.12775 & 0.16 & 44 & $\mathrm{C}_{11} \mathrm{H}_{16} \mathrm{~N}$ & 162.12773 & 0.118 & $\mathrm{BE}$ \\
\hline & 162.09135 & 0.40 & 113 & $\mathrm{C}_{10} \mathrm{H}_{12} \mathrm{NO}$ & 162.09134 & 0.054 & DG \\
\hline & 160.11209 & 0.33 & 95 & $\mathrm{C}_{11} \mathrm{H}_{14} \mathrm{~N}$ & 160.11208 & 0.077 & $\mathrm{BE}$ \\
\hline \multirow[t]{3}{*}{$*$} & 158.09643 & 0.07 & 19 & $\mathrm{C}_{11} \mathrm{H}_{12} \mathrm{~N}$ & 158.09643 & 0.000 & $\mathrm{BE}$ \\
\hline & 146.09643 & 0.18 & 50 & $\mathrm{C}_{10} \mathrm{H}_{12} \mathrm{~N}$ & 146.09643 & 0.012 & BCE \\
\hline & 145.08859 & 0.05 & 13 & $\mathrm{C}_{10} \mathrm{H}_{11} \mathrm{~N}$ & 145.08860 & -0.052 & \\
\hline \multirow[t]{3}{*}{$*$} & 134.09643 & 0.07 & 18 & $\mathrm{C}_{9} \mathrm{H}_{12} \mathrm{~N}$ & 134.09643 & 0.000 & $\mathrm{DE}$ \\
\hline & & & & & $\begin{array}{l}\text { Standard } \\
\text { Deviation }\end{array}$ & 0.093 & \\
\hline & & & & & $\begin{array}{l}\text { Absolute } \\
\text { Average }\end{array}$ & 0.062 & \\
\hline
\end{tabular}


Table S 15: Table showing the percentage of even and odd electron fragments for both fragmentation methods (CAD and EID)

\begin{tabular}{|c|c|c|c|c|}
\hline & \multicolumn{2}{|c|}{ CAD } & \multicolumn{2}{|c|}{ EID } \\
\hline & $\begin{array}{c}\text { Even } \\
\text { Electron } \\
\text { Fragments } \\
\text { (\%) }\end{array}$ & $\begin{array}{c}\text { Odd } \\
\text { Electron } \\
\text { Fragments } \\
(\%)\end{array}$ & $\begin{array}{c}\text { Even } \\
\text { Electron } \\
\text { Fragments } \\
\text { (\%) }\end{array}$ & $\begin{array}{c}\text { Odd } \\
\text { Electron } \\
\text { Fragments } \\
\text { (\%) }\end{array}$ \\
\hline Azoxystrobin & 95.24 & 4.76 & 73.96 & 26.04 \\
\hline Fenpropidin & 85.71 & 14.29 & 55.56 & 44.44 \\
\hline Fluazifop acid & 76.47 & 23.53 & 72.73 & 27.27 \\
\hline Fluazifop-p-butyl & 85.71 & 14.29 & 60.87 & 39.13 \\
\hline Nicosulfuron & 93.33 & 6.67 & 84.62 & 15.38 \\
\hline $\begin{array}{c}\text { Pirimiphos- } \\
\text { methyl }\end{array}$ & 30.00 & 70.00 & 41.33 & 58.67 \\
\hline S-metolachlor & 93.75 & 6.25 & 85.71 & 14.29 \\
\hline
\end{tabular}


a) $\mathrm{CAD}$

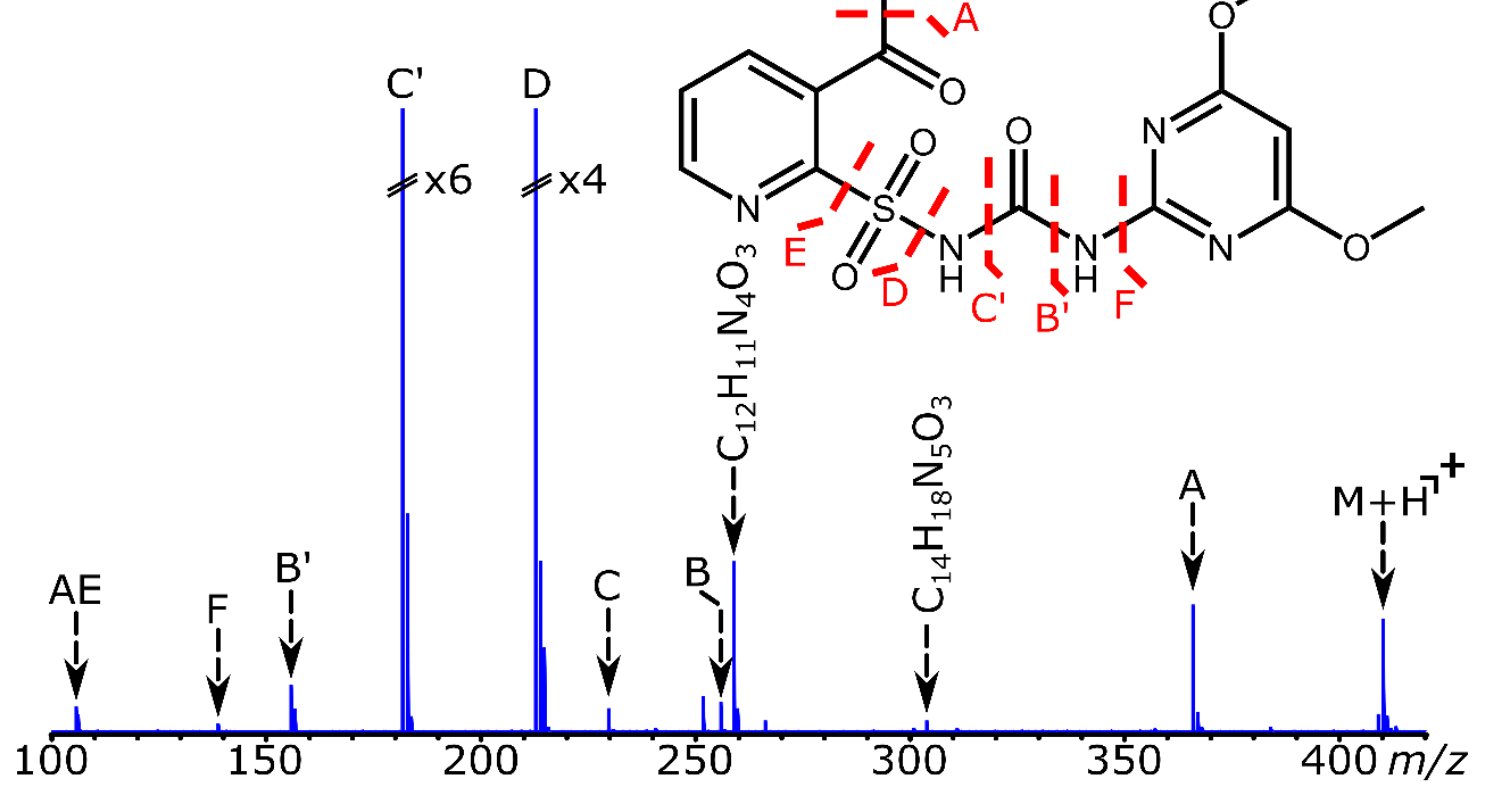

b) EID

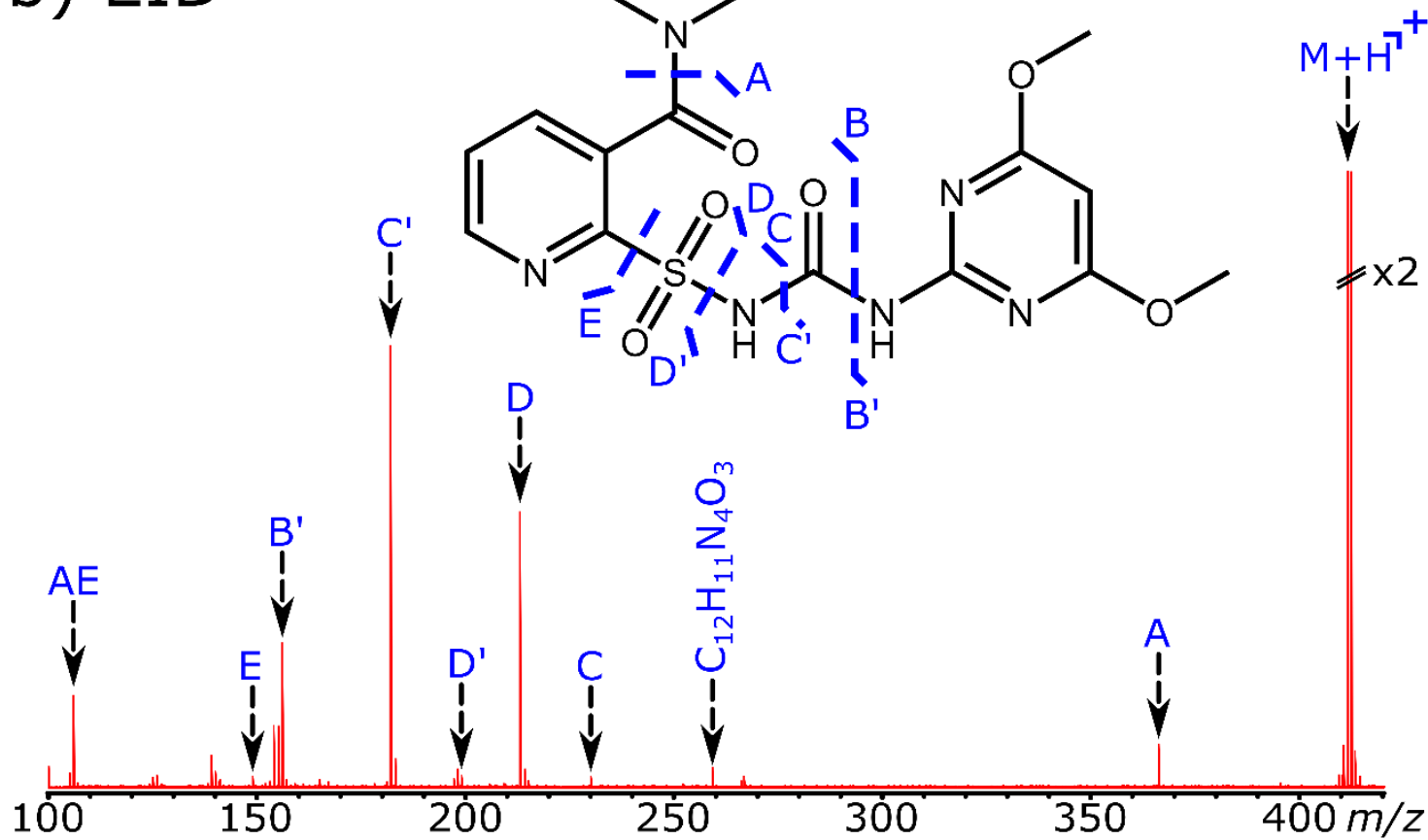

Figure S 1: The fragmentation spectra of nicosulfuron along with the corresponding cleavage diagram via a) CAD (20 V) and b) EID (18 V). Peak assignment tables are displayed in Table S9 \& S10. 


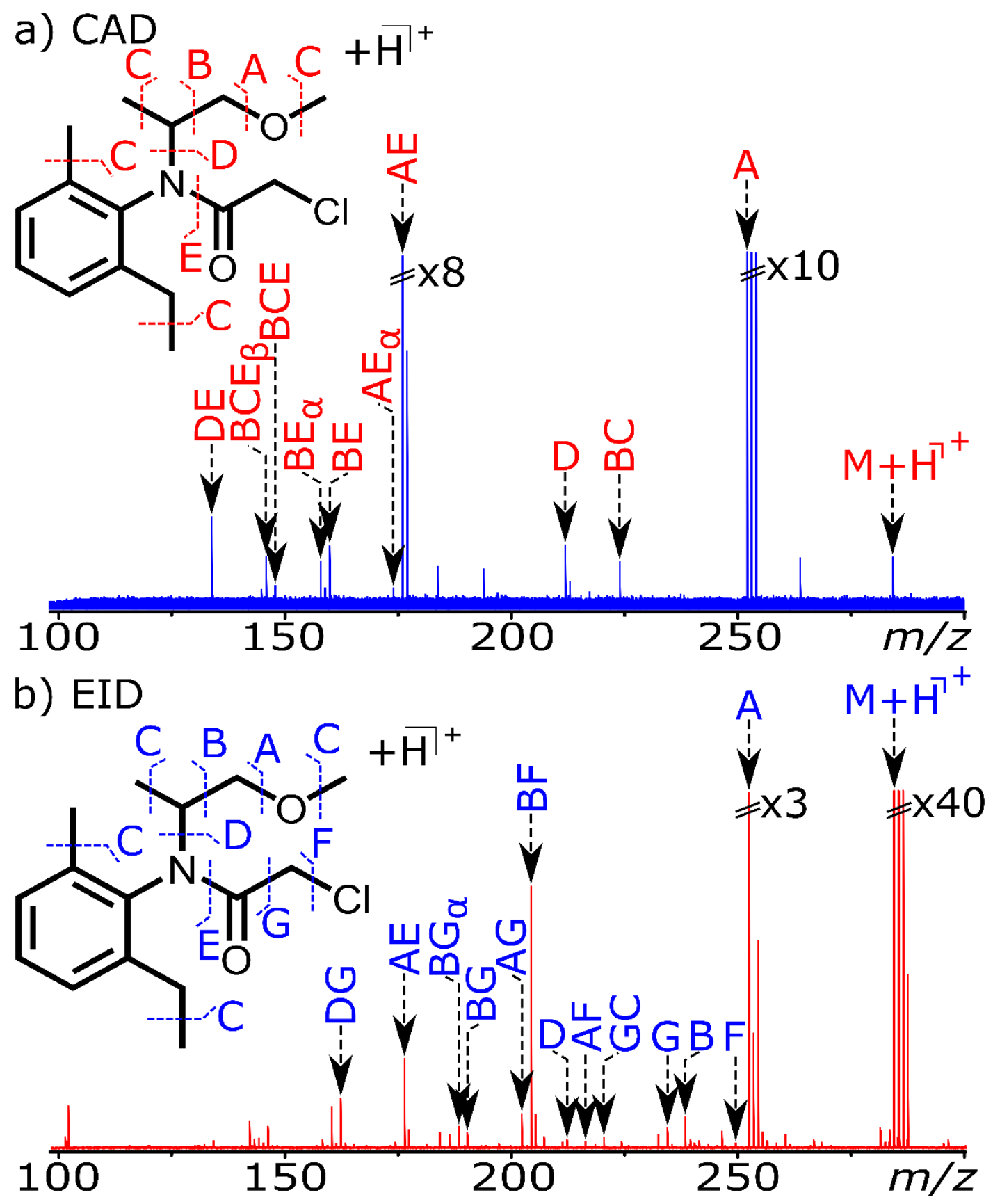

Figure S 2: The fragmentation spectra of s-metolachlor with its corresponding cleavage assignment diagram via a) CAD (20 V) and b) EID (18 V). Peak assignment are listed in Tables S13 \& S14. 


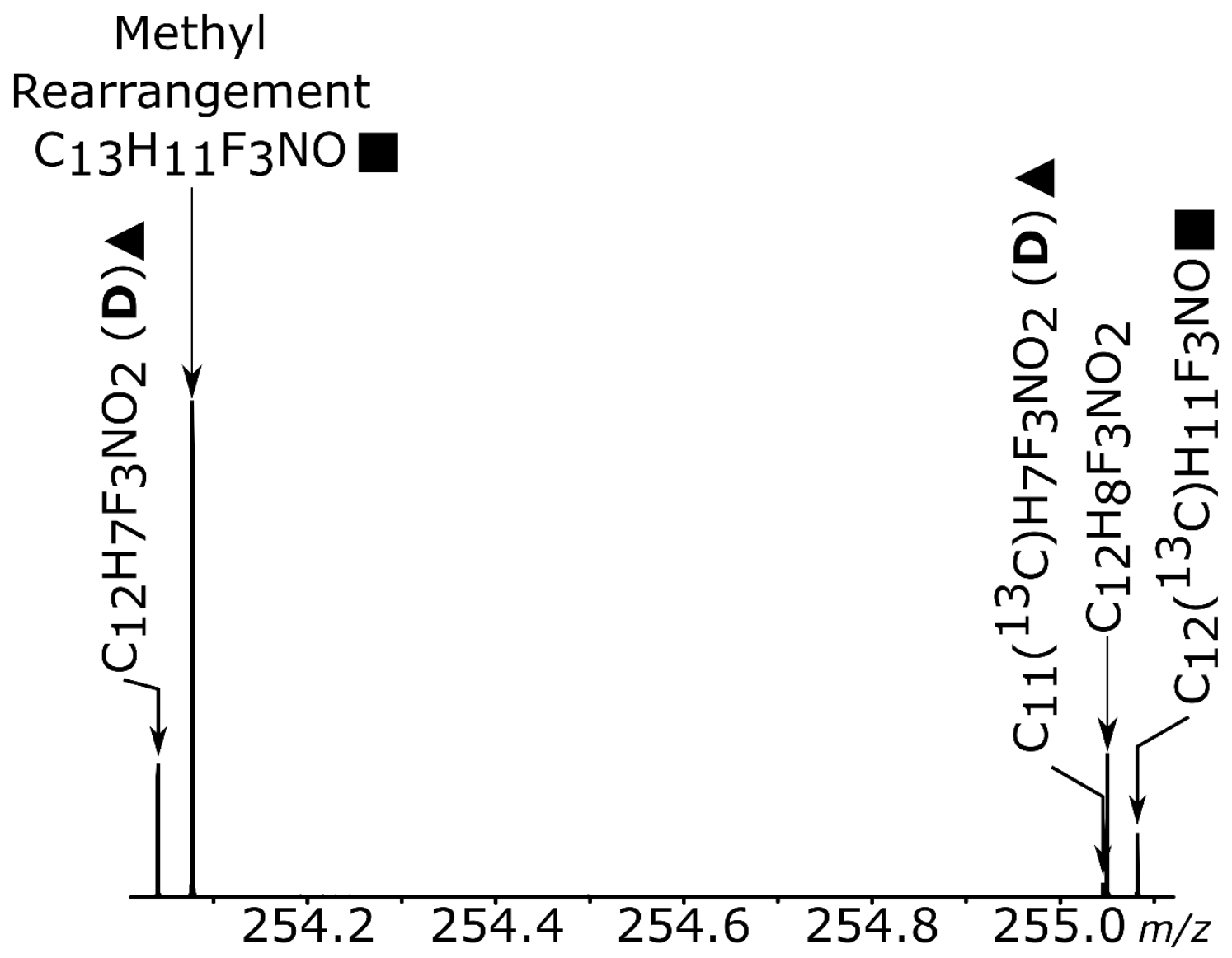

Figure S 3: Zoom in view of the fluazifop acid CAD spectrum showing the separation of the D cleavage $\left(\mathrm{C}_{12} \mathrm{H}_{7} \mathrm{~F}_{3} \mathrm{NO}_{2}\right)$ and the methyl rearrangement $\left(\mathrm{C}_{13} \mathrm{H}_{11} \mathrm{~F}_{3} \mathrm{NO}\right)$ peaks observed at nominal mass of $\mathrm{m} / \mathrm{z} 254$ and their corresponding isotope peaks labelled with a triangle and a square, respectively. Also in this zoomed in spectrum, separation of the isotope peaks and a peak corresponding to a composition of $\mathrm{C}_{12} \mathrm{H}_{8} \mathrm{~F}_{3} \mathrm{NO}_{2}$ can be observed. 
A

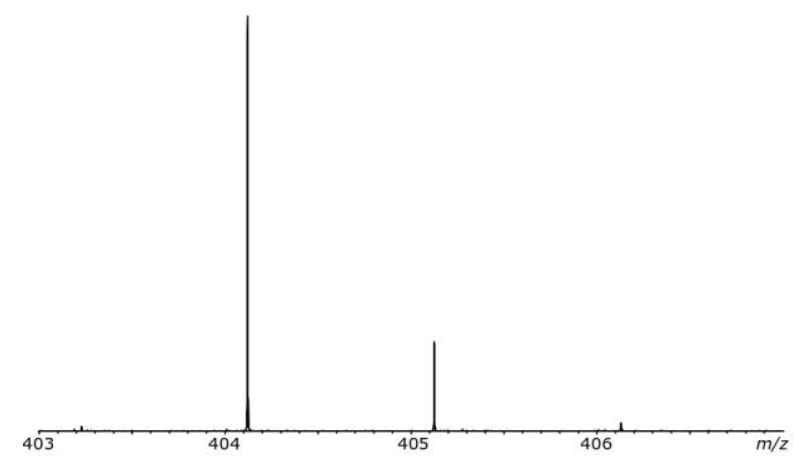

C

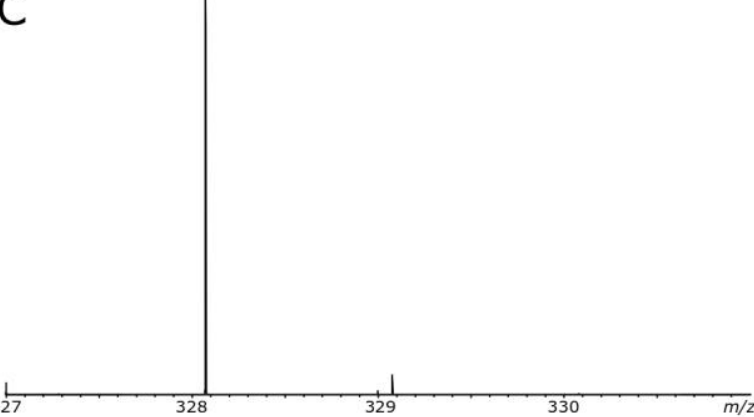

E

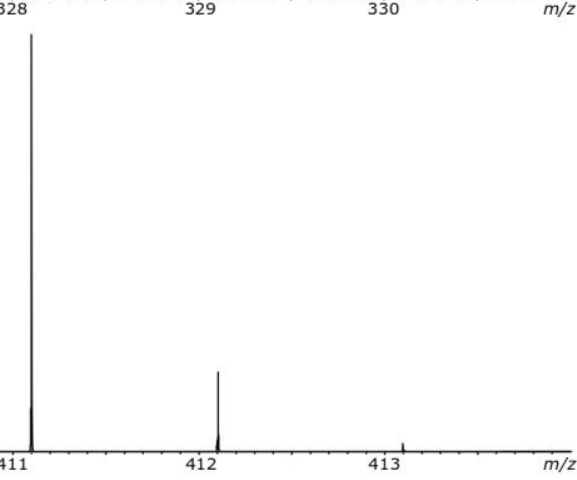

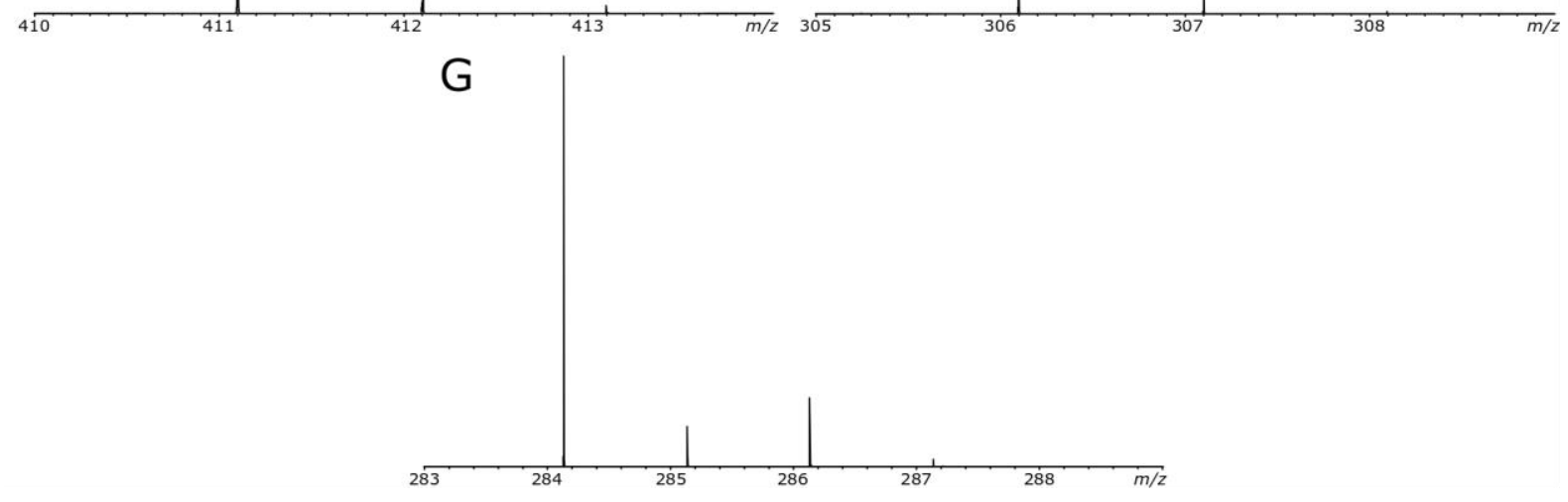

B
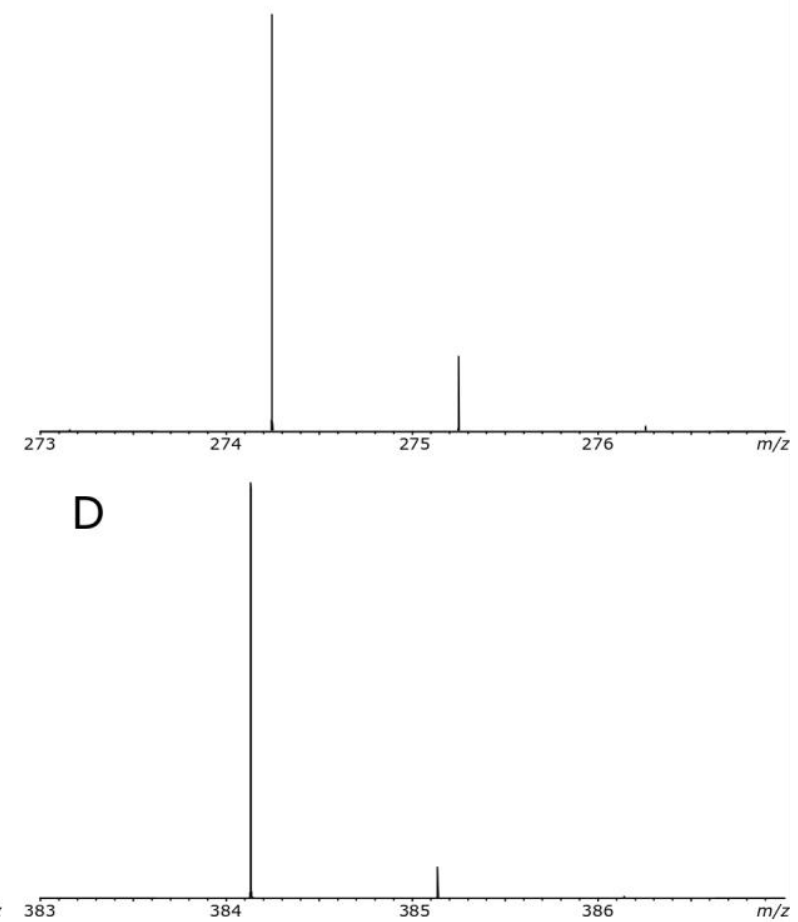

F

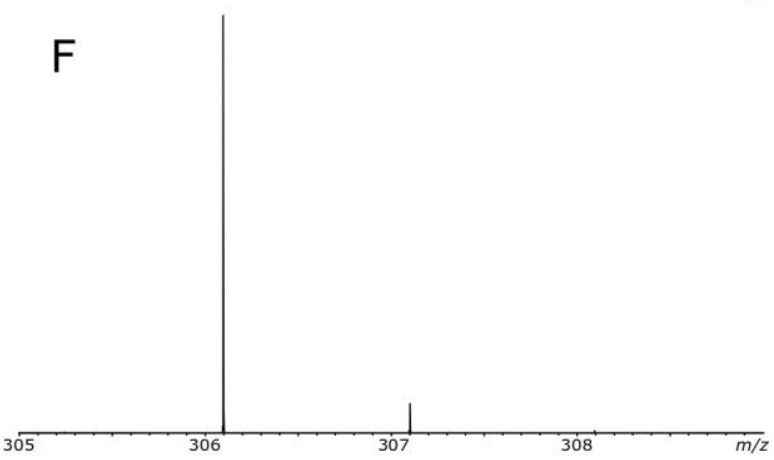

Figure S 4: Isolation spectra for the 7 agrochemicals: A) azoxystrobin, B) fenpropidin, C) fluazifop acid, D) fluazifop-p-butyl, E) nicosulfuron, F) pirimiphos-methyl, and G) S-metolachlor. 